\title{
Bacher and Goudsmit Theory of Complex Spectra
}

\author{
R. E. Trees
}

\begin{abstract}
The Bacher and Goudsmit theory expresses the energy of a state of an atom in terms of a linear combination of energies of states of other ions of the same atom. This theory is tested in the spectra with $d$ - and s-electrons and general conclusions drawn about its accuracy.

In deriving their theory, Bacher and Goudsmit formally introduced many-particle interactions. They showed (to a limited extent) that this was equivalent to a second procedure in which the zero-order wave functions were assumed to be independent of the ionization and successive orders of perturbations were included.

Recently it has been shown that a linear theory, which is a combination of the Slater theory and an empirically derived correction, predicts the separations of terms in any one configuration with about the same accuracy as the Bacher and Goudsmit theory; in this theory, the wave functions are allowed to depend on the degree of ionization. It is shown that the Bacher and Goudsmit theory can be interpreted in a third way that is consistent with this linear theory.

In the light of this latter interpretation, Racah has suggested an empirical modification of the Bacher and Goudsmit theory that often leads to better results; this suggestion has been incorporated in the present paper.
\end{abstract}

\section{Introduction}

Bacher and Goudsmit have shown that it is possible to express the energy levels of an atom correctly to second order by taking linear combinations of the energy levels of the ions of that atom. ${ }^{1}$ They gave explicit formulas for these linear combinations for atoms containing $s$ - and $p$-electrons, and tested the theory in the spectra of oxygen, nitrogen, and carbon. The theory usually predicted the absolute term values with an error less than $2500 \mathrm{~K}^{2}{ }^{2}$ Separations of terms in a given configuration were often given very much better, sometimes within a few hundred kaysers.

In section 2 the theory of Bacher and Goudsmit is derived briefly from a new viewpoint. Bacher and Goudsmit derived their formulas by formally introducing many-body interactions; no explicit form was given for these interactions. They were able to show that in the first and second approximations this was equivalent to using zero-order wave functions that were independent of the degree of ionization, and, with these, making first- and second-order perturbation calculations of the energy, respectively. In the present paper, a third derivation is given, in which the zero-order wave functions of the Slater theory are allowed to vary regularly with the degree of ionization; an explicit two-body interaction is also introduced. This interpretation is justified by the observation that there is a smooth variation of the one-particle and two-particle parameters in a linear theory. ${ }^{3}$ The more specific and easily understood meaning of this interpretation in terms of a linear

\footnotetext{
R. F. Bacher and S. Goudsmit, Phys. Rev. 46, 948 (1934); referred to in the present paper as "BG"

2 "Kayser" is the name adopted by the Joint Commission for Spectroscopy for the unit of wave number, $\mathrm{cm}^{-1}$, and is abbreviated " $\mathrm{K}$ "

3 R. E. Trees and M. M. Harvey, J. Research NBS 49, 397 (1952) RP2378. In section 1 of RP2378 the literature is reviewed, and the methods of analyzing spectra with the Slater theory, for including effects of configuration interaction, and of making allowance for polarization energy with the $L(L+1)$ correction are also outlined and applied. In referring to a "linear theory", the $L(L+1)$ correction is combined with the slater theory, and the radial integrals are regarded as parameters.
}

theory is given in section 5 ; it is equivalent to assuming that the parameters in different ions of the same atom are given by series expansions in powers of the degree of ionization. It is shown that the $\mathrm{BG} n$th approximation accounts for terms up to and including the $n$th power for the one-particle parameters and terms to the $(n-1)$ th power for the two-particle parameters.

In section 3 a few formulas applicable to terms in $d^{n}, d^{n} s$, and $d^{n} s^{2}$ configurations are obtained by using tables of coefficients of fractional parentage and recoupling transformations given by Racah. ${ }^{4}$ In section 4 an application of these formulas is made in the spectra of titanium, vanadium, chromium, and zirconium. It is found that separations of terms in the same configuration are given very closely (the error is less than $400 \mathrm{~K}$ ). Separations of terms in different configurations may be given very poorly, however, as an error of $6000 \mathrm{~K}$ is found in titanium $(Z=22)$. The latter type of error is found to decrease very rapidly in spectra of more highly ionized atoms: in chromium spectra $(Z=24)$ the error is only $600 \mathrm{~K}$, one-tenth the error found for the related titanium spectra. The error is also found to decrease for spectra with higher nuclear charge as it is only $2000 \mathrm{~K}$ in zirconium $(Z=40)$.

Racah has pointed out that a power-series expansion, of the type described previously, converges more rapidly if spectra are chosen having the same degree of ionization. A comparison with experiment, using this empirical modification of the original theory of Bacher and Goudsmit, is also given in section 4.

As indicated previously, the Bacher and Goudsmit theory can be interpreted in terms of a linear theory in which the radial parameters are given by series expansions in the degree of ionization. Checking these expansions is the easiest way to check all the data against the Bacher and Goudsmit theory. The

${ }^{4}$ G. Racah, Phys. Rev. 62, 438 (1942); 63, 367 (1943). These papers are referred to herein as II and III, respectively. 
conclusions reached from the direct application of the theory already cited above, have been verified by using all available data with this method. The detailed calculations needed for this verification are omitted for brevity.

In section 6 the effect of weak configuration interaction in which one electron is excited is considered, and in section 7 the effect of irrationality of the energy eigenvalues is analyzed. The purpose is to illustrate in more detail the direct application of the theory of BG and to indicate where a direct application might lead to results that differ from those obtainable with a linear theory. Such differences are probably too small to be detected within the accuracy obtainable at present with any theory, but more complete comparisons of theory with the presently available experimental data are needed to verify this conclusion.

\section{Bacher and Goudsmit Theory}

\subsection{General Theory}

In this section is given a new derivation of the theory in which it is assumed that the interaction parameters vary smoothly. The Hamiltonian of the $n$-electron system is taken as

where

$$
H=H_{1}+H_{2},
$$

and

$$
H_{1}=\sum_{i=1}^{n}\left(\frac{1}{2 \mu} p_{i}^{2}-\frac{Z e^{2}}{r_{i}}\right)
$$

$$
H_{2}=\sum_{i>j=1}^{n} \frac{e^{2}}{r_{i j}} .
$$

$H_{1}$ and $H_{2}$ are examples of what are called "typeone" and "type-two" terms, respectively, $\left(H_{1}\right.$ is a sum of functions, each depending on the coordinates of a single electron, and $H_{2}$ is a sum of functions for two electrons). A particular selection $P$ of $r$ of the $n$-electrons will be indicated by $P_{r}$. The sum of the Hamiltonians $H\left(P_{r}\right)$ of the $r$-electron systems for all possible choices $P$ is a symmetric function of the $n$-electrons, and the $n$-electron Hamiltonian $H$ is expressible identically as a linear combination of such symmetric sums for two or more values of $r$,

$$
H=\sum C_{r} \sum_{p} H\left(P_{r}\right)
$$

The conditions that this be an identity are the following:

$$
\sum_{r_{\text {ats }}} C_{r}\left(\begin{array}{l}
n \\
r
\end{array}\right)\left(\begin{array}{l}
r \\
1
\end{array}\right)=\left(\begin{array}{l}
n \\
1
\end{array}\right) \text { and } \sum_{r} C_{r}\left(\begin{array}{l}
n \\
r
\end{array}\right)\left(\begin{array}{l}
r \\
2
\end{array}\right)=\left(\begin{array}{l}
n \\
2
\end{array}\right) .
$$

In general, $\left(\begin{array}{l}a \\ b\end{array}\right)$ is the binomial coefficient when $a \geq b \geq 0$,

$$
\left(\begin{array}{l}
a \\
b
\end{array}\right)=\frac{a !}{(a-b) ! b !}
$$

and zero for $b<0$ or $a<b$. The values of the coefficients $C_{r}$ will be indeterminate if there are more than two nonzero $C_{r}$-values. The method of choosing best values of the $C_{r}$ in such cases will be considered below.

The eigenfunction $\psi(\gamma)$ of the $n$-electron system is assumed to have in its specification a definite configuration $l_{1}, l_{2}, \ldots, l_{n}$, and $P_{r}$ is specified by $r$ of these $l$-values; the electrons are regarded as distinguishable, even though some may be equivalent. The electrons in the selection $P_{r}$ have allowed eigenfunctions $\psi(\alpha)$ of the $r$-electron Hamiltonians $H\left(P_{\tau}\right)$; the residual $(n-r)$ electrons have allowed eigenfunctions $\psi(\beta)$ of the $(n-r)$-electron Hamiltonian. The $l$-values of the electrons are included in the specifications $\alpha, \beta$, and $\gamma$, along with $S$-and $L$-values for the combined system and any other quantum numbers needed to specify the coupling. ${ }^{5}$ The function formed by vector coupling the $(n-r)$-electron eigenfunction to the $r$-electron eigenfunction is indicated by $\psi(\alpha, \beta, \gamma)$. A linear combination of thes e functions can be formed that will be identical with $\psi(\gamma)$; this was first pointed out by Bacher and Goudsmit,

$$
\psi(\gamma)=\sum_{\alpha \beta}(\alpha \beta, \gamma \rrbracket \gamma) \psi(\alpha, \beta, \gamma) .
$$

$(\alpha, \beta, \gamma \rrbracket \gamma)$ is called the coefficient of fractional parentage. The calculation of the coefficients of fractional parentage is a mathematical problem that has been considered in detail by Racah. He has tabulated these coefficients for configurations of equivalent $p$ and $d$-electrons in III; their use will be illustrated in section 3 .

A partial energy $E(\alpha)$ of the choice $P_{r}$ is introduced that is associated with the $r$-electrons while they are part of the $n$-electron system and combined to form the state $\alpha$. This energy cannot be observed, and must be approximated from the observed energy of the $r$-electron ion of the atom in the state $\alpha$, which is defined as $W(\alpha)$. The average energy of the choice $P_{r}$ when present in the original $n$-electron configuration $\gamma$ is determined with the help of (4),

$$
\bar{E}\left(\gamma ; P_{\tau}\right)=\sum_{\alpha \beta}(\alpha \beta, \gamma \rrbracket \gamma)^{2} E(\alpha) .
$$

The corresponding approximate energy can be calculated from the observed energies in the $r$-electron ion,

$$
\bar{W}\left(\gamma ; P_{r}\right)=\sum_{\alpha \beta}(\alpha \beta, \gamma \rrbracket \gamma)^{2} W(\alpha) .
$$

The energy of the $n$-electron system is then given identically by an expression similar to (2),

$$
E(\gamma)=\sum_{r} C_{r} \sum_{p} \bar{E}\left(\gamma ; P_{r}\right)
$$

${ }^{5}$ There may be more than one term allowed with a given (SL)-value in the configuration considered (the configuration may be that contained in $\alpha, \beta$ or $\gamma$ ). In such cases the function obtained by a legitimate coupling procedure will not be an eigenfunction of the Hamiltonian, even though it has an allowed symmetry, if it mixes with other allowed eigenfunctions in that configuration having the same $(S L)$-value. In such cases a purely formal difficulty arises because the characterization by parentage is indeterminate, and the thecry given by Bacher and Goudsmit must be amplified by more or less arbitrary additions. In sections 2,3 , and 4 of the present paper these cases are omitted. These omitted cases are considered in sections 5 and 7. 
The corresponding approximate energy is calculated from" the approximate average energies (6),

$$
W(\gamma)=\sum_{r} C_{r} \sum_{p} \bar{W}\left(\gamma ; P_{r}\right) .
$$

The energies $W$ and $\bar{W}$ used above are the same as the $W$ used in $\mathrm{BG}$ for both expressions. Quantities such as $W(\alpha), W(\alpha, \beta)$, etc., on the right side of eq (7) to (14) in BG are sometimes energies of observed spectral terms, but in general they are linear combinations of the observed energies; in the present notation they would be replaced by $\bar{W}\left(\gamma ; P_{1}\right), \bar{W}\left(\gamma ; P_{2}\right)$, etc. Directly observed energies are indicated by $W$, as in the right-hand side of (25) and (26) of BG.

The difference between the exact value (7) and the approximate value (8) is estimated by separating the $E$ 's and $W$ 's into their type-one and type-two parts, and by assuming that the following expansions can be made:

$$
\begin{aligned}
& W_{1}(\alpha)=E_{1}(\alpha)\left\{1+a_{1}(\gamma)(n-r)+a_{2}(\gamma)(n-r)^{2}+\ldots\right\} \\
& W_{2}(\alpha)=E_{2}(\alpha)\left\{1+b_{1}(\gamma)(n-r)+b_{2}(\gamma)(n-r)^{2}+\ldots\right\} .
\end{aligned}
$$

The coefficient $a_{t}(\gamma)$ in (9) will be eliminated from the difference between (7) and (8) if

$$
\sum_{r=1}^{n-1} C_{r}(n-r)^{t}\left(\begin{array}{l}
n \\
r
\end{array}\right)\left(\begin{array}{l}
r \\
1
\end{array}\right)=0
$$

and the coefficient $b_{t}(\gamma)$ will be eliminated if

$$
\sum_{r=1}^{n-1} C_{r}(n-r)^{t}\left(\begin{array}{l}
n \\
r
\end{array}\right)\left(\begin{array}{l}
r \\
2
\end{array}\right)=0 .
$$

A choice of $C_{r}$-values defines an approximation of the same order as the largest value of $t$ for which (10) is satisfied (it is assumed that (10) and (11) are satisfied for all smaller values of $t$ and that relations (3) are satisfied). For instance, in a second approximation, the type-one energies are expanded in a series containing powers up to and including the squares of $(n-r)$, whereas the type-two energies are expanded only to first power in $(n-r)$. Formulas (3), (10), and (11) are not independent. They may be combined into a definition that is easily shown to be equivalent to that just given, by requiring the coefficients $C_{r}$ to satisfy the following $(m+1)$ equations in order to define an $m$ th approximation

$$
\sum_{r=1}^{n-1} C_{r}(n-r)^{t}\left(\begin{array}{l}
n \\
r
\end{array}\right)\left(\begin{array}{l}
r \\
1
\end{array}\right)=\delta_{t 0}\left(\begin{array}{l}
n \\
1
\end{array}\right) \quad(t=0,1, \ldots, m) .
$$

In the derivation of $\mathrm{BG}$, many-body interactions are formally introduced. According to the present notation, this is equivalent to considering not only type-one and type-two terms as being present in (1) but also type-three, type-four, etc., terms. This leads immediately, by generalizing (3), to the definition of the $m^{\text {th }}$ approximation given by Bacher and
Goudsmit. According to their definition the $C_{r^{-}}$ values satisfy the following $(m+1)$ equations:

$$
\sum_{r=1}^{n-1} C_{r}\left(\begin{array}{l}
n \\
r
\end{array}\right)\left(\begin{array}{l}
r \\
k
\end{array}\right)=\left(\begin{array}{l}
n \\
k
\end{array}\right) \quad k=1,2, \ldots,(m+1) .
$$

To show the definitions (12) and (13) are equivalent, the following expansion is utilized for powers $t>0$ of an integer $p:{ }^{6}$

$$
p^{t}=\sum_{\kappa=1}^{t} \alpha_{\kappa}(t)\left(\begin{array}{l}
p \\
\kappa
\end{array}\right)
$$

where

$$
\alpha_{\kappa}(t)=\kappa^{t}-\kappa(\kappa-1)^{t}+\frac{\kappa(\kappa-1)(\kappa-2)^{t}}{2 !}-\cdots
$$

Inserting (14) in (12) leads to the result

$n \sum_{\kappa=1}^{t} \alpha_{\kappa}(t)\left(\begin{array}{c}n-1 \\ \kappa\end{array}\right) \sum_{r=1}^{n-1} C_{r}\left(\begin{array}{l}n-\kappa-1 \\ r-1\end{array}\right)=0 \quad(t=1, \ldots, m)$.

Equation (13) is equivalent to the formula

$$
\sum_{r=1}^{n-1} C_{r}\left(\begin{array}{c}
n-k \\
r-k
\end{array}\right)=1 \quad(k=1,2, \ldots,(m+1)) .
$$

It follows therefore by repeated application of the binominal coefficient relation,

$$
\left(\begin{array}{l}
n-\kappa-1 \\
r-1
\end{array}\right)=\left(\begin{array}{l}
n-\kappa \\
r-1
\end{array}\right)-\left(\begin{array}{l}
n-\kappa-1 \\
r-2
\end{array}\right)
$$

and the use of (13a) that the left side of (12a) vanishes, as it should, whenever (13) is satisfied. When $t=0$ in (12), the equation is the same as (13) when $k=1$, so the equivalence of the two sets of defining equations is demonstrated. A special $m^{\text {th }}$ approximation is defined by the following set of $C_{r^{-}}$ values:

$$
\left.\begin{array}{ll}
C_{r}=(-)^{m-r+1}\left(\begin{array}{c}
n-m-1 \\
m-r+1
\end{array}\right) & {[(r=1,2, \ldots,(m+1))} \\
C_{r}=0 & (r=(m+2), \ldots,(n-1))
\end{array}\right\} .
$$

If there are only two nonzero values of $C_{r}$, and these are $C_{1}$ and $C_{2}$, then they are defined by (15), and relation (8) has the explicit form

$$
W_{1}(\gamma)=\sum_{p} \bar{W}\left(\gamma ; P_{2}\right)-(n-2) \sum_{p} \bar{W}\left(\gamma ; P_{1}\right)
$$

Relations (8) and (12) of BG are special cases of (16), for which $n$ has the values 4 and 5, respectively. As an alternative, it can be assumed that $C_{1}$ and $C_{n-1}$ are the nonzero coefficients, and then the fol-

${ }^{6}$ A treatise on the calculus of finite differences, G. Boole, edited by F. J. Moulton (Macmillan \& Co., London, 1880). 
lowing formula is obtained by solving (12) or (13) (the specification $\gamma$ will be omitted for brevity):

$$
W_{1}(n)=\frac{1}{n-2} \sum_{p} \bar{W}\left(P_{n-1}\right)-\frac{1}{n-2} \sum_{p} \bar{W}\left(P_{1}\right) .
$$

\subsection{The Second Approximation}

If $C_{1}, C_{2}$, and $C_{3}$ are taien as the nonzero coefficients of the second approximation, the following formula is obtained from (15):

$$
\begin{gathered}
W_{2}(n)=\sum_{p} \bar{W}\left(P_{3}\right)-(n-3) \sum_{p} \bar{W}\left(P_{2}\right)+ \\
\frac{1}{2}(n-3)(n-2) \sum_{p} \bar{W}\left(P_{1}\right) .
\end{gathered}
$$

Relations (9) and (13) of BG are special cases of this formula for which $n$ has the values 4 and 5, respectively. In appendix I of BG, perturbation theory was used to show that (18) is equivalent to a calculation that is correct up to and including the second order. In general, reference to "the theory of Bacher and Goudsmit" in the present work applies chiefly to this second approximation.

The complete set of one-electron functions used in appendix $\mathrm{I}$ of $\mathrm{BG}$ was that appropriate to the ionized state of the atom with only a single electron outside of a closed shell. The same zero-order functions were used for the atom in all other states of ionization, and the second-order effect was evaluated with them. This is a poor zero-order approximation for some spectra, ${ }^{7}$ so that "second-order effect," in the sense used by Bacher and Goudsmit, is not synonymous with polarization energy. The latter energy is usually defined as that part of the observed energy that cannot be explained by a calculation in which the one-electron functions are determined by the Hartree-Fock method for each state of ionization. No investigation has been made to determine the variation in accuracy of a secondorder calculation with change of the zero-order eigenfunctions, but Bacher and Goudsmit often obtained poor values in their calculations because the higher-order effects became more important as the zero-order functions became worse approximations.

The influence of polarization on the separation of terms in a single configuration containing $d$ - and $s$-electrons may be represented by a correction proportional to $L(L+1){ }^{8}$ Racah has shown that this correction may be derived by assuming a special type of linearity for the polarization energy, and he has outlined a proof of this assumption of linearity. ${ }^{9}$ The proof is based on the assumption that in the configuration $d^{n}$ the $L(L+1)$ correction arises from

\footnotetext{
7 Similarly defined parameters change by 10 to 30 percent in successive ions when they are evaluated by least squares; they would have the same value if the zero-order functions were the same in all ions. A similar problem is considered by A. Rahman, Physica 19, 377 (1953). See also E. P. Wigner, Phys. Rev. 94, 77 (1954).

8 R. E Trees, Phys, Rev, 84, 1089 (1951).

8 R. E. Trees, Phys. Rev. 84,1089 (1951).
9 G. Racah, Phys. Rev. 85, 381, (1952), and private communications.
}

interactions with configurations $d^{n-2} l^{2}$ and $d^{n-2} l l^{\prime}$ that have two electrons of the original configuration excited. The linear behavior then follows from (54) of BG. Alternatively, it can be demonstrated by comparing (33a) of III with (33c) of III, and assuming that the perturbing configurations are sufficiently far away so that the energy denominators in the second-order perturbation can be assumed constant. The proof shows that the $L(L+1)$ correction is accounted for initially in the first approximation, so that it is on the same footing as the Slater energy. An interaction in which only one electron is excited is included fully for the first time in the second approximation, as shown by (53) and (59) of BG. Any deviation from linear behavior that is produced by such an interaction must be assumed negligible if the $L(L+1)$ correction is used to account for polarization.

In section 4 it is shown that the Bacher and Goudsmit theory leads to close agreement with the observed separation for two terms in the $d^{3} s$ configuration in both titanıum and zirconium, the errors being $357 \mathrm{~K}$ and $293 \mathrm{~K}$, respectively. Bacher and Goudsmit noted that the separation of terms in the same configuration are well predicted in spectra containing $2 s$ - and $2 p$-electrons. ${ }^{10}$ It is concluded that the error of the Bacher and Goudsmit theory in predicting separations of terms in the same configuration will generally be less than $400 \mathrm{~K}$ and that the accuracy is therefore about the same as that obtained with a linear theory.

A large part of the polarization energy is the same for all terms of a given configuration. The energy common to all terms can be investigated with selfconsistent field and other variational methods, but it is not easy to get a reliable picture of the behavior from the few calculations that have been made. One approximation, used fairly often with minor variations, ascribes an energy of 5000 to $10000 \mathrm{~K}$ either to each electron or to each pair of equivalent electrons; interactions between pairs of nonequivalent electrons are neglected in the latter approximation. The energy is independent of $Z$ but depends a little on the types of electrons that are paired. ${ }^{11}$ This part of the polarization energy is very large, and the departures from the described behavior could be appreciable.

The Bacher and Goudsmit theory can be tested in this respect by calculating the separations of terms in different configurations. In section 4 it is shown

10 Tables XVII and XVIII of BG, calculation II. It is noted in BG that agreement better than 200 to $300 \mathrm{~K}$ must be regarded as fortuitous so that the ultimate significant accuracy is regarded as about the same as obtained with the $L(L+1)$ correction. Many separations of terms in the same configuration are predicted with errors greater than $1000 \mathrm{~K}$ in $\mathrm{BG}$, and this must be atributed to the neglect of configuration interaction, or to the fact that the theory is less ac-

curate for spectra with $2 s$ - and $2 p$-electrons. Ycrk, N. Y., 1940). Seitz evaluates energy per electron rather than per pair of equivalent electrons, but his remarks on page 248 justify the latter interpretation. Self-ccnsistent field calculations of $O, O^{+}$, and $\mathrm{O}^{++}$, including exchange and configuration interaction (D. R. Hartree, W' Hartree, B. Swirles, Phil. Trans. Roy. Soc. [A] 238, $229(1939)$ ), support an interpretation based on pairs, a value of 7000 $\mathrm{K}$ being associated with each pair of equivalent $2 p$-electrons. Although a strong dependence on multiplicity is expected (page 250 ), this is shown only to a minor extent in the oxygen calculations; if present, it would be accounted for in the previous consideration of the separations of terms in the same configuration. An additional pcint that should be noted is that comparison of the self-consistent action, indicates that configuration interaction is impcrtant. 
that there is an error of $6000 \mathrm{~K}$ in predicting the energy difference between a term in the $3 d^{4}$ configuration and one in the $3 d^{3} 4 s$ configuration of titanium, and a corresponding error of $2000 \mathrm{~K}$ in similar zirconium spectrum with $4 d$ - and $5 s$-electrons, and also that this error decreases rapidly as ionization increases in the spectra with $3 d$ - and $4 s$-electrons because the spectra of titanium, vanadium, and chromium show errors of 6000,1800 , and $600 \mathrm{~K}$, respectively. Bacher and Goudsmit found an error of about $2000 \mathrm{~K}$ in the spectra with $2 s$ - and $2 p$ electrons; the decrease of the error with increase of $Z$ was slight in these spectra. ${ }^{12}$

The spectra considered here contain four electrons outside of closed shells and are the easiest spectra to analyze with relation (18). A more complete exploration of the theory would consider also spectra with five or more electrons.

\subsection{Higher Approximations}

A third approximation may be obtained from (15), in which $C_{1}, C_{2}, C_{3}$, and $C_{4}$ are nonzero;

$$
\begin{aligned}
W_{3}(n)=\sum_{p} \bar{W}\left(P_{4}\right)-(n-4) \sum_{p} \bar{W}\left(P_{3}\right)+ \\
\frac{(n-4)(n-3)}{2} \sum_{p} \bar{W}\left(P_{2}\right)- \\
\frac{(n-4)(n-3)(n-2)}{3.2} \sum_{p} \bar{W}\left(P_{1}\right) .
\end{aligned}
$$

Relation (14) of BG is a special case of this formula for which $n=5$. Bacher and Goudsmit considered this approximation equivalent to a calculation by perturbation theory correct to thrd order, but they were not able to carry out the complex calculation that would be necessary to demonstrate this rigorously. Separations of terms in spectra of oxygen and nitrogen were calculated a little better in the $W_{3}$ than in the $W_{2}$ approximation. ${ }^{13}$ In section 5 the relative accuracy of $W_{3}$ and $W_{2}$ is discussed from another viewpoint (formulas (56) and (60)), and it is concluded that higher approximations can only account for a small part of the error already noted in the $W_{2}$ approximation.

Any interpretation used to justify the method of Bacher and Goudsmit leads to the conclusion that it is more accurate the more similar the binding of the electrons (and the structure of the configurations) for the different energy levels utilized in the formulas.

12 Table XIV of BG. In discussing this table, Bacher and Goudsmit noted that the errors decreased slightly as $Z$ increased, but from a later discussion it is apparent that they considered this effect of $Z$ as being of minor importance.

${ }_{13}$ Separations of terms in the same configuration were sometimes given with a smaller error by $W_{3}$ than by $W_{2}$, as may be seen by comparing calculation III with calculation II in table XVIII of BG; however, in an almost equal number of eases the agreement was worse. Absolute term values calculated with. $W_{2}$ for the $2 s^{2} p^{3}$ gonfiguration of oxyen were generally in error by less tl on $1000 K$ the $2 s^{2} 2 p^{3}$ configuration of oxygen were generally in error by less than $10 \mathrm{co} K$ (table XIV of BG), whereas the $W_{3}$ approximation indicated term values in the $2 s^{2} 2 p^{4}$ configuration with a larger error, about $1400 \mathrm{~K}$ (table XV of BG). Bacher and Goudsmit note that the $2 s^{2} 2 p^{4}$ configuration is calculated better in the $W_{2}$ approximation, regarding the $s$-electrons as part of the closed shell, than in the $W_{4}$ approximation. The relative errors of absolute term values will naturally decrease for the higher approximation as the absolute value of the energy to the closed shell is much greater.
For example, in calculating term values for $l^{n}$ all configurations specifying a choice of $r$-elections would belong to $l^{r}$. On the other hand, in calculating term values for $l^{n-1} s$ the selection of $r$-elections is specified by either the $l^{r}$ or the $l^{r-1} s$ configuration, so that there are twice as many different configurations to be considered. According to this argument, a given order of approximation would probably predict terms in the $l^{n}$ configuration best, terms in $l^{n-1} s$ next best, and terms in $l^{n-2} s^{2}$ least accurately. This argument has some confirmation which was pointed out by Bacher and Goudsmit. ${ }^{14}$ Bacher and Goudsmit also have noted that the method is inadequate for configurations such as $2 s^{2} 2 p^{2} 4 s$, in which the total energy of the excited electron is less than the electrostatic energy of the inner ones. In such cases, even the separations of terms in the same configurations would not be given accurately. Similiarly, the method gives poor predictions for negative ions. ${ }^{15}$

\section{Formulas for Spectra With $d$ - and s-Electrons}

\subsection{The $1^{n}$ Configuration}

When the configuration specified in $\gamma$ is $l^{n}$, equation (6) has the form

$$
\begin{array}{r}
\bar{W}\left(l^{n} \gamma S L ; l^{r}\right)=\sum_{\alpha_{1} S_{1} L_{1} \alpha_{2} S_{2} L_{2}}\left(l^{r}\left(\alpha_{1} S_{1} L_{1}\right) l^{n-\tau}\left(\alpha_{2} S_{2} L_{2}\right),\right. \\
\left.S L] l^{n} \gamma S L\right)^{2} W\left(l^{r} \alpha_{1} S_{1} L_{1}\right) .
\end{array}
$$

If this relation is used in (17) for $\bar{W}\left(P_{n-1}\right)$, the sum over $P$ introduces a factor $\left(\begin{array}{l}n \\ r\end{array}\right)=\left(\begin{array}{l}n \\ n-1\end{array}\right)=n$, and $(17)$ becomes,

$$
\begin{gathered}
W_{1}\left(l^{n} \gamma S L\right)=\frac{n}{n-2} \sum_{\alpha_{1} S_{1} L_{1}}\left(l^{n-1}\left(\alpha_{1} S_{1} L_{1}\right) l S L \rrbracket l^{n} \gamma S L\right)^{2} \\
\cdot W\left(l^{n-1} \alpha_{1} S_{1} L_{1}\right)-\frac{n}{n-2} W(l)
\end{gathered}
$$

The first term on the right defines the separation of terms in the $l^{n}$ configuration; it has the same form as a relation given by Racah for calculating matrix elements of a general scalar operator $G$ defined between pairs of interacting electrons. ${ }^{16}$ Similiarly, by utilizing (20) with $r=2$, relation (16) becomes

$$
\begin{gathered}
W_{1}\left(l^{n} \gamma S L\right)=\frac{n(n-1)}{2} \sum_{S_{1} L_{1} \alpha_{2} S_{2} L_{2}}\left(l^{2}\left(S_{1} L_{1}\right) l^{n-2}\left(\alpha_{2} S_{2} L_{2}\right),\right. \\
\left.S L] l^{n} \gamma S L\right)^{2} W\left(l^{2} S_{1} L_{1}\right)-n(n-2) W(l) .
\end{gathered}
$$

14 Table XIV of BG. Absolute term values for the $2 p^{4}$ configuration of oxygen are predicted within a few hundred kaysers, which may be the best significant agreement obtainable. Errors for $2 p^{3} 2 s$ are about $1000 \mathrm{~K}$, and they are about $2000 \mathrm{~K}$ for $2 s^{2} 2 p^{2}$.

${ }_{15}$ In footnote 7 of $\mathrm{BG}$ it is noted that the calculated electron affinity of oxygen is $8000 \mathrm{~K}$ and that the observed value is $18000 \mathrm{~K}$. The discontinuity in binding is $8000 \mathrm{~K}$ and that the observed value is $18000 \mathrm{~K}$. The discontinuity in binding
energy of negative icns has been discussed by Ta-You W u in Phys. Rev. 89, 629 (1952).

16 G. Racah, Phys. Rev. 76, 1349 (1949). Formula (1). 
This is the same as an alternative formula given by Racah for calculating matrix elements of a scalar operator. ${ }^{17}$

It is noted in III that the formula corresponding to (22) needs very long calculations and is of practical use only in a few particular cases. Although the direct use of (20) is impractical, except when $r=n-1$ (or in the trivial case where $r$ is unity), a step-by-step procedure, starting with $r=n-1$ and decreasing $r$ a unit at a time, is generally practical. With this procedure, only tables of the coefficients $\left(l^{n-1}\right.$ $\left.\left(\alpha_{1} S_{1} L_{1}\right) l, S L \rrbracket l^{n} \gamma S L\right)$ are needed. These coefficients are defined by (9) III and (11) III, and the coefficients have been tabulated in III for $p^{n}$ and $d^{n}$ configurations.

As an example, the second approximation (18) will be evaluated in detail for a $d^{4}{ }^{5} \mathrm{D}$ term by using (20) in a step-by-step procedure. By using table III of III, relation (20) becomes

$$
\bar{W}\left(d^{4}{ }^{5} \mathrm{D} ; d^{3}\right)=3 / 10 W\left(d^{3}{ }^{4} \mathbf{P}\right)+7 / 10 W\left(d^{3}{ }^{4} \mathrm{~F}\right) .
$$

The $W$ 's on the right are formally evaluated with (20), by using table II of III:

$$
\begin{gathered}
\bar{W}\left(d^{3}{ }^{4} \mathrm{P} ; d^{2}\right)=8 / 15 W\left(d^{2}{ }^{3} \mathrm{P}\right)+7 / 15 W\left(d^{2}{ }^{3} \mathrm{~F}\right), \\
\bar{W}\left(d^{3}{ }^{4} \mathrm{~F} ; d^{2}\right)=1 / 5 W\left(d^{2}{ }^{3} \mathrm{P}\right)+4 / 5 W\left(d^{2}{ }^{3} \mathrm{~F}\right) .
\end{gathered}
$$

These results are then substituted back in (23):

$$
\bar{W}\left(d^{4}{ }^{5} \mathrm{D} ; d^{2}\right)=3 / 10 W\left(d^{2}{ }^{3} \mathrm{P}\right)+7 / 10 W\left(d^{2}{ }^{3} \mathrm{~F}\right) .
$$

Alternately, (26) could have been obtained directly from (20) (with $\mathrm{r}=2$ ) by first calculating the coefficients of fractional parentage $\left(d^{2}\left(S_{1} L_{1}\right) d^{2}\left(S_{2} L_{2}\right)\right.$, $\left.{ }^{5} \mathrm{D} I d^{4} \mathrm{D}\right)$ with $(32)$ of III. The step-by-step procedure used above is simpler because the quantum numbers $\alpha_{2} S_{2} L_{2}$ do not enter the calculation explicitly. The values (23) and (26) are now inserted into (18) and the sums over $P$ replaced by factors $\left(\begin{array}{l}n \\ r\end{array}\right)$ to obtain the desired formula,

$$
\begin{aligned}
W_{2}\left(d^{4}{ }^{5} \mathrm{D}\right) & =6 / 5 W\left(d^{3}{ }^{4} \mathrm{P}\right)+14 / 5 W\left(d^{3}{ }^{4} \mathrm{~F}\right) \\
& -9 / 5 W\left(d^{2}{ }^{3} \mathrm{P}\right)-21 / 5 W\left(d^{2}{ }^{3} \mathrm{~F}\right)+4 W(d) .
\end{aligned}
$$

Formula (27) is a good one to use in testing the Bacher and Goudsmit theory. All term values used in the formula should be unperturbed, and they are likely to be known experimentally. The following two formulas, obtained in a similar fashion, are not so generally useful: the terms that are most likely to be perturbed by configuration interaction

\footnotetext{
17 Formula (33a) of III. To get the expressions to agree exactly, the following generalization of formula (29) of III is used:

$\left(l^{r}\left(\alpha_{1} S_{1} L_{1}\right) l^{n-r}\left(\alpha_{2} S_{2} L_{2}\right) S L l l^{n} \gamma S L\right)$

are starred:

$$
\begin{aligned}
& W_{2}\left(d^{4}{ }^{1} \mathrm{I}\right)=6 / 5 W\left(d^{3}{ }^{2} \mathrm{G}\right) *+14 / 5 W\left(d^{3}{ }^{2} \mathrm{H}\right) \\
& -3 / 7 W\left(d^{2}{ }^{1} \mathrm{D}\right) *-3 W\left(d^{2}{ }^{3} \mathrm{~F}\right)-18 / 7 W\left(d^{2}{ }^{1} \mathrm{G}\right)+4 W(d)
\end{aligned}
$$

$$
\begin{gathered}
W_{2}\left(d^{4}{ }^{3} \mathrm{H}\right) *=1 / 3 W\left(d^{3}{ }^{2} \mathrm{~F}\right) *+4 / 3 W\left(d^{3}{ }^{4} \mathrm{~F}\right) \\
+3 / 5 W\left(d^{3}{ }^{2} \mathrm{G}\right) *+26 / 15 W\left(d^{3}{ }^{2} \mathrm{H}\right) \\
-3 / 5 W\left(d^{2}{ }^{3} \mathrm{P}\right)-2 / 7 W\left(d^{2}{ }^{1} \mathrm{D}\right) *-17 / 5 W\left(d^{2}{ }^{3} \mathrm{~F}\right) * \\
-12 / 7 W\left(d^{2}{ }^{1} \mathrm{G}\right)+4 W(d) .
\end{gathered}
$$

Formulas for all other terms would require an addition to the theory of Bacher and Goudsmit. The parentages of duplicated terms in $d^{4}$ and/or $d^{3}$ would depend on the particular spectrum under consideration, whereas in the formulas for the terms given above the coefficients were defined by purely mathematical considerations.

\subsection{The $l^{n-1} l^{\prime}$ Configuration}

The evaluation of $W\left(\gamma ; P_{r}\right)$ when the specification $\gamma$ contains the $l^{n-1} l^{\prime}$ configuration, can be carried out similarly if tables of coefficients of fractional parentage for nonequivalent electrons are available. ${ }^{18}$ In general, such tables are not available, and the coefficients must be calculated from tables for equivalent electrons with the help of transformations between different coupling schemes given in section 2 of III. Again a step-by-step procedure is simplest, so that only selections $P$ with $n-1$ electrons need be considered. The parent term in the $l^{n-1}$ configuration again is assumed, for simplicity, to be in an exactly specified state. The single selection containing the equivalent electrons has an average energy $\bar{W}\left(\left(l^{n-1} \gamma S L\right) l^{\prime} S^{\prime} L^{\prime} ; l^{n-1}\right)$, which is the same as the observed energy in the ion $W\left(l^{n-1} \gamma S L\right)$. The only new feature is the selection specified by the $l^{n-2} l^{\prime}$ configuration. The coefficients of fractional parentage will have the form

$$
\begin{gathered}
\left.\left(l^{n-2}\left(\alpha S_{1} L_{1}\right) l^{\prime}\left(S^{\prime \prime} L^{\prime \prime}\right), l S^{\prime} L^{\prime}\right] l^{n-1}(\gamma S L) l^{\prime} S^{\prime} L^{\prime}\right) \\
=\left(l^{n-2}\left(\alpha S_{1} L_{1}\right) l S L \rrbracket l^{n-1} \gamma S L\right) \\
\left(S_{1} L_{1} l(S L) l^{\prime} S^{\prime} L^{\prime} \mid S_{1} L_{1} l^{\prime}\left(S^{\prime \prime} L^{\prime \prime}\right) l S^{\prime} L^{\prime}\right) .
\end{gathered}
$$

On the right, the first factor is the coefficient of fractional parentage for equivalent electrons; the

18 Tables for $p^{2} s, p^{3} s$, and $d^{2} p$ have been given by S. Meshkov in Phys. Rev. 91, 871 (1953). With his convention for the normalization of the coefficients, the sums over $P$ required in the formulas given here would be replaced by factors $\left(\begin{array}{l}n \\ r\end{array}\right)$; the different selections $P$ are then accounted for during the calculation of the tables. To allow what seems to be the most direct use of Racah's tables, the coefficients of fractional parentage have been normalized to unity for each selection in the present paper. 
second factor is a transformation coefficient evaluated in (5) of III,

$$
\begin{aligned}
\left(S_{1} L_{1} l(S L) l^{\prime} S^{\prime} L^{\prime} \mid S_{1} L_{1} l^{\prime}\left(S^{\prime \prime} L^{\prime \prime}\right) l S^{\prime} L^{\prime}\right) & \\
= & {\left[(2 S+1)\left(2 S^{\prime \prime}+1\right)(2 L+1)\left(2 L^{\prime \prime}+1\right)\right]^{\frac{1}{2}} } \\
& W\left(S_{\frac{1}{2}}^{\frac{1}{2}} S^{\prime \prime} ; S^{\prime} S_{1}\right) W\left(L l^{\prime} l L^{\prime \prime} ; L^{\prime} L_{1}\right) .
\end{aligned}
$$

The $\mathrm{W}$-functions in this expression are calculated from (36) of II; some values of the W-function have also been tabulated. ${ }^{19}$

The particular case where $l^{\prime}$ is an s-electron is the only one we consider in detail. In this case, the result of applying (30) and (31) reduces to a fairly simple formulation. To evaluate the average energies for a term $l^{n-1}(\gamma S L) s S^{\prime} L$ :

(a) Evaluate $\bar{W}\left(l^{n-1} \gamma S L ; l^{n-2}\right)$ in terms of the observed energies $W\left(l^{n-2} \alpha S_{1} L_{1}\right)$, as already described.

(b) If the spin $S_{1}$ of the ion term in $l^{n-2}$ does not have the same value $S^{\prime}$ as the original term under consideration in $l^{n-1} s$, then add an $s$-electron to this ion term, making the resultant spin $S^{\prime \prime}$ equal to $S$, the spin of the parent term in $l^{n-1} ; 1$. e., change $W\left(l^{n-2} \alpha S_{1} L_{1}\right)$ to $W\left(l^{n-2}\left(\alpha S_{1} L_{1}\right) s S L_{1}\right)$.

(c) If the spin $S_{1}$ has the value $S^{\prime}$, then add the s-electron to the ion, and split the latter into two terms, each having one of the two spins that are allowed, i. e., give $S^{\prime \prime}$ the two values $S$ and $\left(2 S^{\prime}-S\right)$. The part having the resultant spin $\mathrm{S}$ is given a coefficient $\left(2 S^{\prime}+1\right)^{-2}$, whereas the other part has a coefficient of unity minus this; i. e., change $W\left(l^{n-2}\right.$ $\left.\alpha S_{1} L_{1}\right)$ into $\left[\left(2 \dot{S}^{\prime}+1\right)^{-2} W\left(l^{n-2}\left(\alpha S^{\prime} L_{1}\right) s S L_{1}\right)+\lceil 1-\right.$ $\left.\left.\left(2 S^{\prime}+1\right)^{-2}\right] W\left(l^{n-2}\left(\alpha S^{\prime} L_{1}\right) s\left(2 S^{\prime}-S\right) L_{1}\right)\right]$.

This formulation could also be used when $l^{\prime} \neq 0$ to take care of the spin. Another subdivision would then be made among the $L$-values, using only that part of (31) that depends on the orbital momentum.

As an example, the second approximation (18) will be evaluated for a $d^{3}\left({ }^{4} \mathrm{~F}\right) s{ }^{5} \mathrm{~F}$ term. Step (a) is already carried out in (25), so that according to step (b)

$$
\begin{aligned}
& \bar{W}\left(d^{3}\left({ }^{4} \mathrm{~F}\right) s{ }^{5} \mathrm{~F} ; d^{2} s\right) \\
& \quad=1 / 5 W\left(d^{2}\left({ }^{3} \mathrm{P}\right) s{ }^{4} \mathrm{P}\right)+4 / 5 W\left(d^{2}\left({ }^{3} \mathrm{~F}\right) s{ }^{4} \mathrm{~F}\right) .
\end{aligned}
$$

The procedure is repeated for the terms on the right, and the results obtained are substituted back in (32) to get the average energy for the $d s$ selection:

$$
\bar{W}\left(d^{3}\left({ }^{4} \mathbf{F}\right) s{ }^{5} \mathbf{F} ; d s\right)=W\left(d s{ }^{3} \mathrm{D}\right) .
$$

This result could also be written by inspection. The average energies (32), (33), and (25) are inserted into (18), together with the trivial values for $\bar{W}\left(\gamma ; d^{3}\right), \bar{W}(\gamma ; d)$, and $\bar{W}(\gamma ; s)$; the sums over $P$ intro-

\footnotetext{
${ }^{10}$ L. C. Biedenharn, Oak Ridge National Laboratory Report ORNL-1098 (unpublished).
}

duce factors $\left(\begin{array}{c}n-1 \\ r\end{array}\right)$ when $P_{r}$ is the $l^{r}$ configuration and $\left(\begin{array}{c}n-1 \\ r-1\end{array}\right)$ when $P_{\tau}$ is the $l^{r-1} s$ configuration:

$$
\begin{gathered}
W_{2}\left(d^{3}\left({ }^{4} \mathrm{~F}\right) s^{5} \mathrm{~F}\right)=W\left(d^{3}{ }^{4} \mathrm{~F}\right)+3 / 5 W\left(d^{2} s{ }^{4} \mathrm{P}\right) \\
+12 / 5 W\left(d^{2} s{ }^{4} \mathrm{~F}\right)-3 / 5 W\left(d^{2}{ }^{3} \mathrm{P}\right) \\
-12 / 5 W\left(d^{2}{ }^{3} \mathrm{~F}\right)-3 W\left(d s^{3} \mathrm{D}\right)+3 W(d)+W(s) .
\end{gathered}
$$

The following formulas are obtained similarly:

$$
\begin{array}{r}
W_{2}\left(d^{3}\left({ }^{4} \mathrm{P}\right) s^{5} \mathrm{P}\right)=W\left(d^{3} \mathrm{P}\right)+8 / 5 W\left(d^{2} s^{4} \mathrm{P}\right) \\
+7 / 5 W\left(d^{2} s{ }^{4} \mathrm{~F}\right)-8 / 5 W\left(d^{2}{ }^{3} \mathrm{P}\right)-7 / 5 W\left(d^{2}{ }^{3} \mathrm{~F}\right) \\
-3 W\left(d s^{3} \mathrm{D}\right)+3 W(d)+W(s)
\end{array}
$$

$W_{2}\left(d^{3}\left({ }^{2} \mathrm{H}\right) s^{1} \mathrm{H}\right)=W\left(d^{3}{ }^{2} \mathrm{H}\right)+3 / 2 W\left(d^{2} s{ }^{2} \mathrm{~F}\right) *$

$$
\begin{gathered}
+3 / 2 W\left(d^{2} s^{2} \mathrm{G}\right) *-3 / 2 W\left(d^{2}{ }^{3} \mathrm{~F}\right)-3 / 2 W\left(d^{2}{ }^{1} \mathrm{G}\right) \\
-3 / 2 W\left(d s^{3} \mathrm{D}\right)-3 / 2 W\left(d s{ }^{1} \mathrm{D}\right) * \\
+3 W(d)+W(s),
\end{gathered}
$$

$$
\begin{array}{r}
W_{2}\left(d^{3}\left({ }^{2} \mathrm{H}\right) s^{3} \mathrm{H}\right)^{*}=W\left(d^{3}{ }^{2} \mathrm{H}\right)+1 / 6 W\left(d^{2} s{ }^{2} \mathrm{~F}\right) * \\
+4 / 3 W\left(d^{2} s{ }^{4} \mathrm{~F}\right)+3 / 2 W\left(d^{2} s^{2} \mathrm{G}\right) *-3 / 2 W\left(d^{2}{ }^{3} \mathrm{~F}\right) \\
-3 / 2 W\left(d^{2}{ }^{1} \mathrm{G}\right)-1 / 2 W\left(d s{ }^{1} \mathrm{D}\right) *-5 / 2 W\left(d s^{3} \mathrm{D}\right) \\
+3 W(d)+W(s)
\end{array}
$$

Formulas (34) and (35) are valid even when configuration interaction is present.

\subsection{The $l^{n-2} l^{\prime 2}$ Configuration}

The final configuration considered is $l^{n-2} l^{\prime 2}$. Both the $l^{n-2}$ and the $l^{\prime 2}$ configuration are assumed to have $S L$-values that are exactly specified. For the selection $P_{n-1}$ that contains only one of the $l^{\prime}$-electrons, the coefficient of fractional parentage is simply a transformation coefficient given by (6) of III:

$$
\begin{gathered}
\left.\left(l^{n-2}(\gamma S L) l^{\prime} S_{1} L_{1}, l^{\prime} S^{\prime \prime} L^{\prime \prime}\right] l^{n-2}(\gamma S L) l^{\prime 2}\left(S^{\prime} L^{\prime}\right) S^{\prime \prime} L^{\prime \prime}\right) \\
=\left(S L, l^{\prime 2}\left(S^{\prime} L^{\prime}\right) S^{\prime \prime} L^{\prime \prime} \mid S L l^{\prime}\left(S_{1} L_{1}\right) l^{\prime} S^{\prime \prime} L^{\prime \prime}\right) \\
=\left[\left(2 S^{\prime}+1\right)\left(2 S_{1}+1\right)\left(2 L^{\prime}+1\right)\left(2 L_{1}+1\right)\right]^{\frac{1}{2}} \\
W\left(S \frac{1}{2} S^{\prime \prime} \frac{1}{2} ; S_{1} S^{\prime}\right) W\left(L l^{\prime} L^{\prime \prime} l^{\prime} ; L_{1} L^{\prime}\right) .
\end{gathered}
$$

This leads to a simple formulation for the $l^{n-2} s^{2}$ configuration: (d) The parentage of an $s$-electron in a term $l^{n-2} s^{2} \gamma S L$ is divided in the ratio of the multiplicities between the possible parent terms, generally two. 
For the selection $P_{n-1}$ that contains both l' electrons, the coefficient of fractional parentage becomes,

$$
\begin{gathered}
\left(l^{n-3}\left(\alpha S_{1} L_{1}\right) l^{\prime 2}\left(S^{\prime} L^{\prime}\right) S_{2} L_{2} l S^{\prime \prime} L^{\prime \prime} \rrbracket l^{n-2}(\gamma S L) l^{\prime 2}\left(S^{\prime} L^{\prime}\right) S^{\prime \prime} L^{\prime \prime}\right) \\
=\left(l^{n-3}\left(\alpha S_{1} L_{1}\right) l S L \rrbracket l^{n-2} \gamma S L\right)
\end{gathered}
$$$$
\left(S_{1} L_{1} l(S L) S^{\prime} L^{\prime}\left(S^{\prime \prime} L^{\prime \prime}\right) \mid S_{1} L_{1} S^{\prime} L^{\prime}\left(S_{2} L_{2}\right) l S^{\prime \prime} L^{\prime \prime}\right) .
$$

The transformation coefficient is given by (5) of III and has a form similar to (31). In a term $l^{n-2} s^{2} \gamma S L$, however, the closed shell of two $s$-electrons can be disregarded for this selection, so that (39) reduces to the procedure already given for a configuration of equivalent electrons.

To evaluate the average energy (6) for a $d^{2} s^{2}{ }^{3} \mathrm{~F}$ term the formulation (d) is used:

$$
\begin{gathered}
\bar{W}\left(d^{2} s^{2}{ }^{3} \mathrm{~F} ; d^{2} s\right)=2 / 3 W\left(d^{2} s^{4} \mathrm{~F}\right)+1 / 3 W\left(d^{2} s^{2} \mathrm{~F}\right), \\
\bar{W}\left(d^{2} s^{2}{ }^{3} \mathrm{~F} ; d s^{2}\right)=W\left(d s^{2} \mathrm{D}\right) .
\end{gathered}
$$

Relation (41) is obtained by inspection; using (d) again with (41) leads to the average energy for a choice $d s$,

$$
\bar{W}\left(d^{2} s^{2}{ }^{3} \mathrm{~F} ; d s\right)=3 / 4 W\left(d s^{3} \mathrm{D}\right)+1 / 4 W\left(d s^{1} \mathrm{D}\right) .
$$

The result (42) could be obtained also from (40) by use of formulations (b) and (c),

$$
\begin{gathered}
\bar{W}\left(d^{2} s{ }^{4} \mathrm{~F} ; d s\right)=W\left(d s{ }^{3} \mathrm{D}\right), \\
\bar{W}\left(d^{2} s{ }^{2} \mathrm{~F} ; d s\right)=1 / 4 W\left(d s^{3} \mathrm{D}\right)+3 / 4 W\left(d s{ }^{1} \mathrm{D}\right) .
\end{gathered}
$$

When these values are substituted for the observed energies $W$ on the right of (40) the result (42) is obtained; this checks the consistency of the procedures and illustrates the use of (c). The values (40), (41), and (42), together with trivial values for $\bar{W}\left(\gamma ; d^{2}\right)$, $\bar{W}\left(\gamma ; s^{2}\right), \bar{W}(\gamma ; d)$, and $\bar{W}(\gamma ; s)$ are then inserted into (18). The number of selections $P_{r}$ of type $l^{r}$ is $\left(\begin{array}{c}n-2 \\ r\end{array}\right)$; $l^{r-1} s$ can be selected in $2\left(\begin{array}{c}n-2 \\ r-1\end{array}\right)$ ways and $7^{r-2} s^{2}$ in $\left(\begin{array}{l}n-2 \\ r-2\end{array}\right)$ ways:

$$
\begin{gathered}
W_{2}\left(d^{2} s^{2}{ }^{3} \mathrm{~F}\right) *=4 / 3 W\left(d^{2} s^{4} \mathrm{~F}\right)+2 / 3 W\left(d^{2} s^{2} \mathrm{~F}\right) * \\
+2 W\left(d s^{2}{ }^{2} \mathrm{D}\right)^{*}-3 W\left(d s^{3} \mathrm{D}\right) \\
-W\left(d s^{1} \mathrm{D}\right) *-W\left(d^{2}{ }^{3} \mathrm{~F}\right) \\
-W\left(s^{2}{ }^{1} \mathrm{~S}\right) *+2 W(d)+2 W(s) . \quad(44)
\end{gathered}
$$

The following formula is obtained in the same way

$$
\begin{gathered}
W_{2}\left(d^{2} s^{2}{ }^{3} \mathrm{P}\right) *=4 / 3 W\left(d^{2} s^{4} \mathrm{P}\right)+2 / 3 W\left(d^{2} s{ }^{2} \mathrm{P}\right) * \\
+2 W\left(d s^{2}{ }^{2} \mathrm{D}\right) *-3 W\left(d s^{3} \mathrm{D}\right) \\
-W\left(d s^{1} \mathrm{D}\right) *-W\left(d^{2}{ }^{1} \mathrm{G}\right) \\
-W\left(s^{2}{ }^{1} \mathrm{~S}\right) *+2 W(d)+2 W(s) .
\end{gathered}
$$

The formulas for the $d^{2} s^{2}$ configuration always contain terms that are likely to be perturbed by configuration interaction. The $s^{2}{ }^{1} \mathrm{~S}$ term is usually unknown, and the applicability of the formulas is limited by this also.

\section{Comparison With Experiment}

In all the formulas derived above, the observed energies, $W$, are referred to the core with all four electrons removed. Because ionization potentials are often not accurate, these absolute energies may not be well known. To check the theory of Bacher and Goudsmit only differences between terms have been calculated. Ionization potentials cancel in the differences, and energies $W$ can then be taken as the relative-energy levels with respect to the lowest term as zero; these are given in a Bureau Circular. ${ }^{20}$ In particular, the following two differences would be unaffected by configuration interaction:

$$
\begin{gathered}
D_{1} \equiv W_{2}\left(d^{3} s{ }^{5} \mathrm{P}\right)-W_{2}\left(d^{3} s{ }^{5} \mathrm{~F}\right)=W\left(d^{3}{ }^{4} \mathrm{P}\right)-W\left(d^{3}{ }^{4} \mathrm{~F}\right) \\
\quad+W\left(d^{2} s{ }^{4} \mathrm{P}\right)-W\left(d^{2} s{ }^{4} \mathrm{~F}\right)-W\left(d^{2}{ }^{3} \mathrm{P}\right)+W\left(d^{2}{ }^{3} \mathrm{~F}\right)
\end{gathered}
$$

$$
\begin{aligned}
D_{2} & \equiv W_{2}\left(d^{4}{ }^{5} \mathrm{D}\right)-W_{2}\left(d^{3} s{ }^{5} \mathrm{~F}\right)=6 / 5 W\left(d^{3}{ }^{4} \mathrm{P}\right) \\
& +9 / 5 W\left(d^{3}{ }^{4} \mathrm{~F}\right)-3 / 5 W\left(d^{2} s{ }^{4} \mathrm{P}\right)-12 / 5 W\left(d^{2} s{ }^{4} \mathrm{~F}\right) \\
& -6 / 5 W\left(d^{2}{ }^{3} \mathrm{P}\right)-9 / 5 W\left(d^{2}{ }^{3} \mathrm{~F}\right)+3 W\left(d s^{3} \mathrm{D}\right) \\
& +W(d)-W(s) .
\end{aligned}
$$

In table 1 the calculated and observed values of $D_{1}$ and $D_{2}$ are compared. In the spectra of titanium and zirconium sufficient data are available to compare the $D_{1}$-values. The calculated and observed values are in close agreement, the calculated value exceeding the observed value by $357 \mathrm{~K}$ in titanium and by $293 \mathrm{~K}$ in zirconium. Separations of terms in the same configuration are thus given closely by the theory. By assuming that $D_{1}$ will be given with the same error in more highly ionized spectra, it is possible to calculate positions for some terms that are not known experimentally, and use the values obtained to calculate $D_{2}$ in these spectra. ${ }^{21}$ According to table 1 the difference between the two terms in different configurations is predicted very badly by (47) for titanium, the error in $D_{2}$ being $6068 \mathrm{~K}$. However, the error is much less for the spectra of the higher ionization of vanadium (error $1841 \mathrm{~K}$ ), and it is still less in chromium (error $635 \mathrm{~K}$ ). The error is also less in the homologous sequences of zirconium (error $2296 \mathrm{~K}$ ), where the nuclear charge is much greater $(Z=40)$ than in titanium $(Z=22)$, but the degree of ionization is the same. The

${ }^{20}$ C. E. Moore, Atomic energy levels, NBS Circular 467, vol. I (1949), vol. II (1952)

${ }_{21}$ The $d^{2}\left({ }^{3} \mathrm{P}\right){ }^{4} \mathrm{P}$ term is unknown in V III and Cr IV; the calculated positions of these terms are given in table 1 . If the errors in $D_{1}$ were to decrease rapidly as the errors in $D_{2}$ do, then these calculated positions would be about $357 \mathrm{~K}$ too high, and the calculated values of $D_{2}$ would be too small by about three-fifths of this and the calculated values of $D_{2}$ would be too small by about three-fifths of this
error, or $220 \mathrm{~K}$. This is a small error compared to the total errors found in the calculated values of $D_{2}$. However, it is noted later that the errors in $D_{1}$ probably will not decrease. 
TABLE 1. Term separations in spectra with ${ }^{-}$- and s-electrons

$$
\begin{aligned}
& D_{1} \equiv W\left(d^{3} s^{5} \mathrm{P}\right)-W\left(d^{3} s^{5} \mathrm{~F}\right) \\
& D_{2} \equiv W\left(d^{4}{ }^{5} \mathrm{D}\right)-W\left(d^{3} s^{5} \mathrm{~F}\right)
\end{aligned}
$$

\begin{tabular}{|c|c|c|c|c|}
\hline \multirow{2}{*}{ Term } & $\mathrm{Ti}$ & V & $\mathrm{Cr}$ & $\mathrm{Zr}$ \\
\hline & \multicolumn{4}{|c|}{ Observed values } \\
\hline $\begin{array}{l}d^{4}{ }^{5} D \\
d^{3} s \\
d^{5} s \\
{ }^{5} \mathrm{~F}\end{array}$ & $\begin{array}{r}28881 \\
6721 \\
14055\end{array}$ & $\begin{array}{r}206 \\
2926 \\
13617\end{array}$ & $\begin{array}{r}350 \\
50020 \\
63261\end{array}$ & $\begin{array}{r}22138 \\
5460 \\
11103\end{array}$ \\
\hline $\begin{array}{l}D_{1} \\
D_{2}\end{array}$ & $\begin{array}{r}7334 \\
22160\end{array}$ & $\begin{array}{r}10721 \\
-2720\end{array}$ & $\begin{array}{r}13241 \\
-49670\end{array}$ & $\begin{array}{r}5643 \\
16678\end{array}$ \\
\hline $\begin{array}{r}d^{3}{ }^{4} \mathrm{P} \\
d^{3} 4 \mathrm{~F} \\
d^{2} s^{4} \mathrm{P} \\
d^{2} s{ }^{4} \mathrm{~F}\end{array}$ & $\begin{array}{r}9452 \\
1085 \\
9968 \\
225\end{array}$ & $\begin{array}{c}11668 \\
336 \\
(57014) \\
44343\end{array}$ & $\begin{array}{r}14324 \\
554 \\
(119671) \\
104637\end{array}$ & $\begin{array}{r}9824 \\
3273 \\
7860 \\
758\end{array}$ \\
\hline $\begin{array}{r}d^{2}{ }^{3} \mathrm{P} \\
d^{2}{ }^{3} \mathrm{~F} \\
d s^{3} \mathrm{D}\end{array}$ & $\begin{array}{r}10661 \\
242 \\
38277\end{array}$ & $\begin{array}{r}13344 \\
419 \\
96547\end{array}$ & $\begin{array}{r}15868 \\
662 \\
167715\end{array}$ & $\begin{array}{r}8581 \\
864 \\
19063\end{array}$ \\
\hline \multirow[t]{2}{*}{$\begin{array}{l}d^{2} \mathrm{D} \\
s^{2} \mathrm{~S}\end{array}$} & $\begin{array}{r}230 \\
80379\end{array}$ & $\begin{array}{r}372 \\
148100\end{array}$ & $\begin{array}{r}574 \\
227775\end{array}$ & $\begin{array}{r}750 \\
38258\end{array}$ \\
\hline & \multicolumn{4}{|c|}{ Calculated differences } \\
\hline \multirow[t]{2}{*}{$\begin{array}{l}D_{1} \\
D_{2}\end{array}$} & $\begin{array}{r}7691 \\
28228\end{array}$ & $\begin{array}{c}(11078) \\
-879\end{array}$ & $\begin{array}{c}(13598) \\
-49035\end{array}$ & $\begin{array}{r}5936 \\
18974\end{array}$ \\
\hline & \multicolumn{4}{|c|}{ Errors } \\
\hline $\begin{array}{l}D_{1}(\text { calc })-D_{1}(\mathrm{obs}) \\
D_{2}(\text { calc })-D_{2}(\mathrm{obs})\end{array}$ & $\begin{array}{r}357 \\
6068\end{array}$ & $\begin{array}{r}\mathrm{a}(357) \\
1841\end{array}$ & $\begin{array}{l}a(357) \\
\quad 635\end{array}$ & $\begin{array}{r}293 \\
2296\end{array}$ \\
\hline
\end{tabular}

aValues in parentheses are calculated to make the error in $D_{1}$ the same for all ${ }_{\mathrm{s}}$ pectra with $3 d$ - and $4 s$-electrons. improvement from titanium to vanadium to chromium is especially remarkable in view of the fact that the errors in $D_{2}$ form a rapidly decreasing geometric sequence, whereas $D_{2}$ itself starts at a large positive value and decreases to a twice as large negative value.

Racah ${ }^{22}$ has pointed out that the energies would be expected to vary more smoothly if the degree of ionization (rather than the atomic number) were the same in the different spectra. The results obtained by use of this empirical modification of the Bacher and Goudsmit theory are given in table 2. Except for the first spectra, the errors in calculated values of $D_{2}$ are on the order of $100 \mathrm{~K}$; part of the good agreement must be fortuitous, however.

A partial explanation of the poor agreement obtained in the first spectra may be that the $d^{3}{ }^{4} \mathrm{P}$ (of Sc $I$ and $\mathrm{Y}_{\mathrm{I}}$ ) is perturbed by interaction with the term $d s d^{\prime} f^{4} \mathrm{P}$ (in the spectra with $4 d$ - and $5 s$-electrons, this has the added effect of making the calculated position for the $d^{2}{ }^{3} \mathrm{~F}$ too high, as seen from the small separation of the $d^{2}{ }^{3} \mathrm{P}$ and $d^{2}{ }^{3} \mathrm{~F}$ in $\left.\mathrm{Sr} \mathrm{I}\right)$. This effect can be estimated in the spectra containing $3 d$ - and $4 s$-electrons by calculating the position of $d^{3}{ }^{4} \mathrm{P}$ so that the error in $D_{1}$ will be $170 \mathrm{~K}$ as it is in the second spectra. When this calculated position (40048 K) is used, the error in $D_{2}$ is still very large $(-5769 \mathrm{~K})$. Racah has noted that the observed position of $d^{2} \mathrm{D}$ in $\mathrm{K} \mathrm{I}$ is about $5117 \mathrm{~K}$ lower than expected from the behavior of the difference between baricenters of the

22 G. Racah, Bul. Research Council Israel III, 290 (1954). I am indebted to Professor Racah for making a preliminary copy of this manuscript available and for discussing the relationship of his results to those of this paper. See also $\mathbf{M}$ A. Catalan, F. Rohrlich, and A. G. Shenstone, Proc. Roy. Soc. [A] 221, 421 (1954).

\begin{tabular}{|c|c|c|c|c|c|c|c|c|c|c|c|}
\hline \multirow{3}{*}{ Term } & \multicolumn{11}{|c|}{ Spectrum } \\
\hline & \multicolumn{2}{|c|}{ I } & \multicolumn{2}{|c|}{ II } & \multicolumn{2}{|c|}{ III } & IV & \multicolumn{2}{|c|}{ I } & \multicolumn{2}{|c|}{ II } \\
\hline & \multicolumn{11}{|c|}{ Observed values } \\
\hline $\begin{array}{r}d^{4}{ }^{5} \mathrm{D} \\
d^{3} s{ }^{5} \mathrm{~F} \\
d^{3} s{ }^{5} \mathrm{P}\end{array}$ & (Ti) & $\begin{array}{r}28881 \\
6721 \\
14055\end{array}$ & $(\mathrm{~V})$ & $\begin{array}{r}206 \\
2926 \\
13647\end{array}$ & $(\mathrm{Cr})$ & $\begin{array}{r}350 \\
50020 \\
63261\end{array}$ & $\begin{array}{l}537 \\
112296 \\
\end{array}$ & $(\mathrm{Zr})$ & $\begin{array}{r}22138 \\
5460 \\
11103\end{array}$ & $(\mathrm{Nb})$ & $\begin{array}{r}772 \\
3397 \\
11034\end{array}$ \\
\hline $\begin{array}{l}D_{1} \\
D_{2}\end{array}$ & & $\begin{array}{r}7334 \\
222160\end{array}$ & & $\begin{array}{r}10721 \\
-2720\end{array}$ & & $\begin{array}{r}13241 \\
-49670\end{array}$ & -111759 & & $\begin{array}{r}5643 \\
16678\end{array}$ & & $\begin{array}{r}7637 \\
-2625\end{array}$ \\
\hline $\begin{array}{r}d^{3}{ }^{4} \mathrm{P} \\
d^{3}{ }^{4} \mathrm{~F} \\
d^{2} s{ }^{4} \mathrm{P} \\
d^{2} s{ }^{4} \mathrm{~F}\end{array}$ & $(\mathrm{Sc})$ & $\begin{array}{l}36540 \\
33846 \\
17969 \\
11610\end{array}$ & $(\mathrm{Ti})$ & $\begin{array}{r}9452 \\
1085 \\
9968 \\
225\end{array}$ & (V) & $\begin{array}{c}11668 \\
336 \\
(56841) \\
44343\end{array}$ & $\begin{array}{rr}\text { (Cr) } & 14324 \\
554 \\
\mathrm{a} 119671 \\
104637\end{array}$ & $(\mathrm{Y})$ & $\begin{array}{l}32210 \\
29605 \\
15385 \\
11277\end{array}$ & $(\mathrm{Zr})$ & $\begin{array}{r}9824 \\
3273 \\
7860 \\
758\end{array}$ \\
\hline $\begin{array}{l}d^{2}{ }^{3} \mathrm{P} \\
d^{2}{ }^{3} \mathrm{~F} \\
d s^{3} \mathrm{D}\end{array}$ & (Ca) & $\begin{array}{l}48551 \\
43494 \\
20357\end{array}$ & (Sc) & $\begin{array}{r}12128 \\
4909 \\
105\end{array}$ & (Ti) & $\begin{array}{r}10661 \\
242 \\
38277\end{array}$ & $\begin{array}{r}\text { (V) } 13344 \\
419 \\
96547\end{array}$ & $(\mathrm{Sr})$ & $\begin{array}{c}44662 \\
(43989) \\
18254\end{array}$ & $(\mathrm{Y})$ & $\begin{array}{r}14048 \\
8429 \\
1193\end{array}$ \\
\hline \multirow[t]{2}{*}{$\begin{array}{l}d^{2} \mathrm{D} \\
s^{2} \mathrm{~S}\end{array}$} & $(\mathrm{~K})$ & $\begin{array}{r}21535 \\
0\end{array}$ & $(\mathrm{Ca})$ & $\begin{array}{r}13687 \\
0\end{array}$ & (Sc) & $\begin{array}{r}118 \\
25537\end{array}$ & $\begin{array}{lr}(\mathrm{Ti}) & 230 \\
& 80379\end{array}$ & $(\mathrm{Rb})$ & $\begin{array}{r}19355 \\
0\end{array}$ & $(\mathrm{Sr})$ & $\begin{array}{r}14724 \\
0\end{array}$ \\
\hline & \multicolumn{11}{|c|}{ Calculated differences } \\
\hline \multirow[t]{2}{*}{$\begin{array}{l}D_{1} \\
D_{2}\end{array}$} & & $\begin{array}{r}3996 \\
12181\end{array}$ & & $\begin{array}{r}10891 \\
-2613\end{array}$ & & $\begin{array}{c}(13411) \\
-49738\end{array}$ & -112020 & & $\begin{array}{c}(6040) \\
-3012\end{array}$ & & $\begin{array}{r}8034 \\
-2582\end{array}$ \\
\hline & \multicolumn{11}{|c|}{ Errors } \\
\hline $\begin{array}{l}D_{1}(\text { calc })-D_{1}(\text { obs }) \\
D_{2} \text { (calc) }-D_{2}(\text { obs })\end{array}$ & & $\begin{array}{l}-3338 \\
-9979\end{array}$ & & $\begin{array}{l}170 \\
107\end{array}$ & & b $\begin{array}{c}(170) \\
-68\end{array}$ & -261 & & $\begin{array}{c}\text { b (397) } \\
-19690\end{array}$ & & $\begin{array}{r}397 \\
43\end{array}$ \\
\hline
\end{tabular}

TABLE 2. Term separations in spectra with d-and s-electrons

a From table 1 calculation.

b Values in parentheses calculated to make error in $D_{1}$ have the indicated value. 
$d^{n-1} s$ and $d^{n}$ configurations in Ca I, Sc I, Ti I, and $\mathrm{V}$ I, and this may explain the poor agreement obtained in first spectra.

\section{Relations Beiween Radial Parameters}

All formulas of the Bacher and Goudsmit theory can be used also to calculate the matrix elements of scalar operators; this has been noted in specific cases in the discussions of formulas (21) and (22), and it was also pointed out in BG. If the term values could be expressed in terms of such matrix elements, and if the zero-order functions were the same for all configurations, then the formulas derived from (16) to (19) (such as (46) and (47)) would be identities if the $W$ 's were replaced by matrix elements of these operators.

The matrix elements of the operators are linear combinations of a limited number of radial in tegrals. For the Slater theory, these combinations are well known (most of the formulas necessary for the present paper are contained in II). The observed separations of terms in the same configuration are predicted well by the Slater theory (i. e., with an accuracy equal to that obtainable with the Bacher and Goudsmit theory) if an empirical correction proportional to $L(L+1)^{23}$ is added to account for the polarization energy (see footnotes 8 and 9). Radial integrals defined by zero-order one-electron functions having the same $n$ - and $l$-values must be considered independent of each other when in different configurations to get this good agreement, however; if the Bacher and Goudsmit theory were directly applicable, all such integrals would have to have the same value. The derivation of the theory, given in section 2 , shows how much of the observed variation of these radial integrals will be accounted for by the Bacher and Goudsmit theory in any given approximation.

To illustrate, formulas for the $W$ 's that enter into (46) are given here according to the notation used in section 2 :

$$
\begin{aligned}
& W\left(d^{3} s^{5} \mathrm{P}\right)=A\left(d^{3} s\right)-3 G_{2}\left(d^{3} s\right)-16 \alpha\left(d^{3} s\right) \\
& W\left(d^{3} s^{5} \mathrm{~F}\right)=A\left(d^{3} s\right)-15 B\left(d^{3} s\right)-3 G_{2}\left(d^{3} s\right)-6 \alpha\left(d^{3} s\right) \\
& W\left(d^{3} \quad{ }^{4} \mathrm{P}\right)=A\left(d^{3}\right)-16 \alpha\left(d^{3}\right) \\
& W\left(d^{3} \quad{ }^{4} F\right)=A\left(d^{3}\right)-15 B\left(d^{3}\right)-6 \alpha\left(d^{3}\right) \\
& W\left(d^{2} s^{4} \mathrm{P}\right)=A\left(d^{2} s\right)+7 B\left(d^{2} s\right)-2 G_{2}\left(d^{2} s\right)-10 \alpha\left(d^{2} s\right) \\
& W\left(d^{2} s^{4} \mathrm{~F}\right)=A\left(d^{2} s\right)-8 B\left(d^{2} s\right)-2 G_{2}\left(d^{2} s\right) \\
& W\left(d^{2} \quad{ }^{3} \mathrm{P}\right)=A\left(d^{2}\right)+7 B\left(d^{2}\right)-10 \alpha\left(d^{2}\right) \\
& W\left(d^{2}{ }^{3} \mathrm{~F}\right)=A\left(d^{2}\right)-8 B\left(d^{2}\right)
\end{aligned}
$$

${ }^{23}$ The $L(L+1)$ correction is represented as the matrix element of the scalar operator $2 \alpha \sum_{i>j=1}^{n} l_{j} \cdot l_{i}$. The value of this for a term in the $l^{n} g^{k}$ configuration is $\alpha[L(L+1)-n l(l+1)]$. In the present work, this, rather, than simply $\alpha L(L+1)$, is added to each term; this change in the form of the correction is compensated by made so that the relationships obtained later between the $A$-values in different configurations will have a simpler form.
If these values are substituted in (46), then the latter is an identity if the $B^{\prime}$ 's and $\alpha$ 's in different configurations are assumed equal. Relation (46) will still be satisfied if the following two less restrictive conditions hold:

$$
\begin{gathered}
B\left(d^{3} s\right)=B\left(d^{3}\right)+B\left(d^{2} s\right)-B\left(d^{2}\right), \\
\alpha\left(d^{3} s\right)=\alpha\left(d^{3}\right)+\alpha\left(d^{2} s\right)-\alpha\left(d^{2}\right) .
\end{gathered}
$$

It is sufficient for $B-2 / 3 \alpha$ to satisfy a relation of similar form for (46) to be satisfied, but if separations of all pairs of terms in the $d^{3} s$ configuration are to be correct, then both relations must hold and in addition the following two:

$$
\begin{gathered}
C\left(d^{3} s\right)=C\left(d^{3}\right)+C\left(d^{2} s\right)-C\left(d^{2}\right), \\
G_{2}\left(d^{3} s\right)=2 G_{2}\left(d^{2} s\right)-G_{2}(d s) .
\end{gathered}
$$

Formulas of this type can be derived easily by inspection, and there is no need to consider fractional parentage. To obtain the second equation in (50), for instance, note that in (18) the combination of a $d$ - and an $s$-electron (needed to define $G_{2}$ ) occurs three ways in $W_{2}\left(d^{3} s\right)$; it occurs two ways in each of three possible selections $P^{3}$ that contain the $s$-electron (in the $d^{2} s$ configuration); it occurs only once in each of the three possible selections $P_{2}$ that contain the $s$-electron (in the $d s$ configuration). As far as the relation for the parameter $G_{2}$ is concerned, this direct interpretation of (18) leads immediately to (50) (multiplied by a factor of three). As $B, C$, and $\alpha$ are all defined by the combination of two $d$-electrons, a similar derivation leads to the same form of equation for all three of these parameters, and this equation is the one represented by (49) and the first equation of $(50)$. In this way it can be shown that the Bacher and Goudsmit second approximation (relation (18) with $n=4$ ) will predict correctly the separations of terms in the same configuration if the following relations hold between the parameters:

$$
\begin{gathered}
B\left(d^{4}\right)=2 B\left(d^{3}\right)-B\left(d^{2}\right) \\
B\left(d^{3} s\right)=B\left(d^{3}\right)+B\left(d^{2} s\right)-B\left(d^{2}\right) \\
B\left(d^{2} s^{2}\right)=2 B\left(d^{2} s\right)-B\left(d^{2}\right) .
\end{gathered}
$$

Three similar relations must be satisfied by the $C$ 's and three more by the $\alpha$ 's. Also, it is necessary that

$$
G_{2}\left(d^{3} s\right)=2 G_{2}\left(d^{2} s\right)-G_{2}(d s) .
$$

The derivation from (18) fails to bring out the fact that the two-electron parameters must have a linear variation if the formulas are to be valid. In general, a two-electron parameter defined between electrons with orbital angular momentum $l$ and $l^{\prime}$ in the configuration $\gamma$ will be specified by $(\gamma) G\left(l l^{\prime}\right)$. According to the derivation with the second series of (9), only a first-power variation with the number of 
electrons in $\gamma$ can be accounted for by the Bacher and Goudsmit second approximation. In particular then, any one of the parameters $B, C$, or $\alpha$ must satisfy a relation of the form

$$
\left(d^{r-k} s^{k}\right) G(d d)=A+r B+k C .
$$

It is easy to verify that (51) to (53) are satisfied when a formula of this kind is applicable.

It is possible to generalize (55) to include all similar spectra rather than just those of the ions of the same atom, in two-, three- and four-electron spectra. If $q$ is the degree of ionization, this generalization will have the form $\left(d^{r-k} s^{k}\right) G(d d)=A+r R+$ $k C+q D$. The difference $D_{1}$ defined by (46) (and equal to $15 B-10 \alpha$ according to the linear theory) should also be expressible by a formula of this type. Racah ${ }^{22}$ has fitted 34 experimentally observed $P-F$ differences in spectra with $3 d$ - and $4 s$-electrons with a mean error of $340 \mathrm{~K}$ by use of the formula

$$
D_{1}=2679+853 r+1334 k+3158 q-111 q^{2} .
$$

This formula is adjusted to spectra with eight different ionizations, and without a quadratic term the mean error would be increased to about $520 \mathrm{~K}$. According to this formula, the differences $D_{1}$ calculated from (46) will be too great on the average by about $220 \mathrm{~K}$. Modifying the theory so that spectra with the same ionization are utilized tends to eliminate this systematic error. This could also be accomplished by using the $W_{3}$ approximation.

The original series (9) will often have to be satisfied, for a given $\gamma$, by more than one value of $\alpha$, and this leads to the conclusion that the ratios of similarly defined radial parameters in different spectra of a given element should be equal. In particular, the following ratio must be constant in configurations with $d$ - and $s$-electrons:

$$
\frac{B\left(d^{r} s^{k}\right)}{C\left(d^{r} s^{k}\right)}=\text { constant }
$$

This is not really a necessary consequence of the theory developed in section 2 , however, as the series (9) can be reinterpreted in terms of radial parameters that independently satisfy relations such as (55). When irrational roots are considered, it is shown in section 7 that (57) is a necessary condition for consistency of this interpretation, in terms of a linear theory, with a direct application of the method of BG.

In the same way that the differences such as (46) were replaced by the formulas (51) to (54), the differences similar to (47) can be replaced by relationships between the $A$ values. The Bacher and Goudsmit second approximation will give the separations of terms in different configurations correctly if the following two relationships between the $A$ 's are satisfied:

$$
\begin{aligned}
A\left(d^{4}\right)-A\left(d^{3} s\right)=3 A\left(d^{3}\right) & -3 A\left(d^{2} s\right)-3 A\left(d^{2}\right) \\
+ & +3 A(d s)+A(d)-A(s)
\end{aligned}
$$

$$
\begin{array}{r}
A\left(d^{3} s\right)-A\left(d^{2} s^{2}\right)=A\left(d^{3}\right)+A\left(d^{2} s\right)-2 A\left(d s^{2}\right) \\
-2 A\left(d^{2}\right)+A(d s)+A\left(s^{2}\right)+A(d) \\
-A(s)+2 G_{2}\left(d^{2} s\right)-2 G_{2}(d s)
\end{array}
$$

By redefining the $A$-values suitably, ${ }^{24}$ the dependence on $G_{2}$ may be omitted from (59); equations (58) and (59) may therefore be regarded as two relations between the differences $A\left(d^{r-k} s^{k}\right)-A\left(d^{r-k-1} s^{k+1}\right)$. In the second approximation, the parameter $A$ must satisfy a cubic relation, and these differences then satisfy quadratic equations having the general form

$$
A\left(d^{r-k} s^{k}\right)-A\left(d^{r-k-1} s^{k+1}\right)=D+E k+F r+G k r+H r^{2} .
$$

The $k^{2}$ dependence is omitted because it adds nothing extra when $k$ has only the two values 0 and 1 . It is easy to verify that (58) and (59) are satisfied when a formula like this is applicable. This formula can be generalized to apply to all similar spectra in a manner similar to that used for (55) if the " $A$ " is suitably defined in the spectra with more than four electrons. If third (or fourth) power terms were important, a constant (or linear) error would result. The errors of $D_{2}$ in table 1 change too rapidly to be explained in this way, however.

In the one-electron, two-electron, and threeelectron spectra considered in section 4, there are five experimentally determinable differences between $A$-values, and the five unknown constants in (60) can be evaluated from these observed values. Similarly, the three constants in (55) can be evaluated from observed separations of terms in the twoelectron and three-electron spectra. The Bacher and Goudsmit second approximation is then equivalent to using the formulas obtained in this way to extrapolate values in the four-electron system.

This equivalence has been verified by using all available experimental data in titanium and zirconium to evaluate the radial parameters for all spectra with least squares. ${ }^{25}$ The results confirmed

24 The parameter $G_{2}$ appears in (59) because an $s^{2}$ subshell is neglected in defining the $A$-values; thus the formula for a $d^{2} s^{2}{ }^{3} F$ term would have the same form as the last relation in (48). If the effects of the $s$-electrons in the $s^{2}$ subskell were explicitly expressed, as done when only a single s-electron is present, then $2 G_{2}\left(d^{2} s^{2}\right)$ would be subtracted from the formulas for all terms in the $d^{2} s^{2}$ configuration. This has not been done since the effect would be included in $A\left(d^{2} s^{2}\right)$ if the parameters were evaluated by least squares and $G_{2}\left(d^{2} s^{2}\right)$ could not be if the parameters were evaluated by least squares and $G_{2}\left(d^{2} s^{2}\right)$ could not be
evaluated. This applies also to $G_{2}\left(d s^{2}\right)$. The $G$ 's that cannot be evaluated evaluated. This applies also to $G_{2}\left(d s^{2}\right)$. The $G$ 's that cannot be evalu
satisfy the relation $G_{2}\left(d^{2} s^{2}\right)-G_{2}\left(d s^{2}\right)=G_{2}\left(d^{2} s\right)-G_{2}(d s)=G_{2}\left(d^{3} s\right)-G_{2}\left(d^{2} s\right)$.

${ }_{25}$ The $a{ }^{1} \mathrm{~F}$ term of Tir and the $a^{1} \mathrm{~S}$ and $c^{3} \mathrm{~F}$ terms of $\mathrm{Zr}$ I were excluded. The $a^{1} \mathrm{~F}$ term is probably not real. It was predicted $2000 \mathrm{~K}$ lower than observed when it was not included in the least-squares calculation. As long as it was included, it and several other terms were in error by 500 to $600 \mathrm{~K}$, regardless of uncertainties in effects of ecnfiguration interaction. It is not well-established experimentally, as only three weak lines were observed; one strong line used to identify it is a $8000 \mathrm{~K}$ higher the $8000 \mathrm{~K}$ higher than cbserved, as is ter, certain that this term is also unrea!. The $c{ }^{5} \mathrm{~F}$ term was calculated $5000 \mathrm{~K}$ higher than observed; although highly unlikely, it is not certain that consideration of of configuration interaction could not explain the result. However only one strong transition has been observed from each level of this triplet; five other confirming transitions are all weak (C. C. Kiess and H. K. Kiess, BS J. Research 6, 621 (1931) $\mathrm{RP296)}$. The $a^{5} \mathrm{D}, b^{3} C$, and $d^{3} \mathrm{P}$ terms in the $d^{4}$ configuration of Ti $\mathrm{r}$ are observed much closer together than called for by the theory; this may be because cf configuration interaction, or it may represent a failure of the $W_{2}$ approximation. In addition to the experimental values listed in Atomic Energy Levels, the recently located terms $d^{2} \mathrm{D}$ of $\mathrm{Zr}$ II and the $a^{1} \mathrm{~S}$ and $b$ is terms of Zr III (C. C. Kiess, J. Opt. Soc. Am.43, 1024 (1953) )were also available. I am indebted to Dr. Kies for furnishing these data before publication. 
the conclusion already obtained from the limited amount of data utilized in table 1 . In all spectra of both elements, the terms could be fitted with absolute errors less than $400 \mathrm{~K}$. The parameters obtained could be adjusted to fit the relations contained in (51) to (57) without significantly changing the original agreement. This verifies the conclusion that the separations of terms in the same configuration are given by the Bacher and Goudsmit method with an absolute error less than $400 \mathrm{~K}$ in titanium and zirconium. The discrepancy in calculating $A\left(d^{4}\right)-$ $A\left(d^{3} s\right)$ from relation (58) was found to be $6560 \mathrm{~K}$ in titanium and $2086 \mathrm{~K}$ in zirconium. These values are approximately the same as the errors found for $D_{2}$ in table 1 , which verifies the conclusion that calculated separations of terms in different configurations may be considerably in error.

If the ionization potentials are accuratley known the aboslute values of the $A$ 's can be calculated (rather than just the differences (58) and (59)),

$$
\begin{aligned}
& A\left(d^{4}\right)=4 A\left(d^{3}\right)-6 A\left(d^{2}\right)+4 A(d)+\delta \\
A\left(d^{3} s\right)= & A\left(d^{3}\right)+3 A\left(d^{2} s\right)-3 A\left(d^{2}\right)
\end{aligned}
$$$$
-3 A(d s)+A(s)+3 A(d)+\delta
$$

$$
A\left(d^{2} s^{2}\right)=2 A\left(d^{2} s\right)+2 A\left(d s^{2}\right)-A\left(d^{2}\right)-4 A(d s)-A\left(s^{2}\right)
$$

$$
+2 A(d)+2 A(s)-2 G_{2}\left(d^{2} s\right)+2 G_{2}(d s)+\delta,
$$

where

$$
\delta=I_{1}-3 I_{2}+3 I_{3}-I_{4} .
$$

The ionization potentials appear only in the combination $\delta ; I_{1}$ is the principal ionization potention of the four-electron spectrum ( $T \mathrm{i}$ I, for instance); $I_{2}$ is that for the three-electron spectrum (as $T \mathrm{i} \mathrm{II}$ ), etc. If (61) to (63) are utilized, errors in the theory will not cancel as they may in the differences (58) and (59). In titanium the error of (61) in calculating $A\left(d^{4}\right)$ is found to be $24130 \mathrm{~K}$, and the error of $(62)$ in $A\left(d^{3} s\right)$ is $1757 \mathrm{~K}$; on the other hand, the corresponding errors in zirconium are $-2100 \mathrm{~K}$ and -4186 $\mathrm{K}$. The inconsistent behavior of the errors in these two calculations probably arises from the well-known need for better ionization potentials, particularly in the one- and two-electron spectra.

\section{Weak Configuration Interaction}

The proof of the method of Bacher and Goudsmit is established with second-order perturbation theory, so that the theory is not directly applicable if configuration interaction is too strong to be evaluated with perturbation theory. However, it may not be directly applicable even if the spectrum has weak configuration interaction that can be evaluated with perturbation theory.

For example, consider interaction between terms of $d^{n}$ and those of $d^{n-1} s$ and assume that second-order perturbation theory can be used. The perturbation of, say, a $d^{3}{ }^{2} \mathrm{G}$ term by a $d^{2} s^{2} \mathrm{G}$ term is evaluated in terms of a characteristic interaction parameter associated with the $d^{3}$ and $d^{2} s$ configurations; this is designated $H_{2}\left(d^{3}-d^{2} s\right)$ in agreement with the notation in (80) of III. For arbitrary $n$, there is a similar parameter defined between $d^{n}$ and $d^{n-1} s$. Partly with the help of table XX of III the following two perturbations are evaluated:

$$
\begin{aligned}
\Delta\left(d^{3}{ }^{2} \mathrm{G}\right) & =\frac{50\left[H_{2}\left(d^{3}-d^{2} s\right)\right]^{2}}{W\left(d^{3}{ }^{2} \mathrm{G}\right)-W\left(d^{2} s^{2} \mathrm{G}\right)} \\
\Delta\left(d^{21} \mathrm{D}\right) & =\frac{140\left[H_{2}\left(d^{2}-d s\right)\right]^{2}}{W\left(d^{2}{ }^{1} \mathrm{D}\right)-W\left(d s^{1} \mathrm{D}\right)} .
\end{aligned}
$$

By considering terms marked with asterisks in (28), it follows that configuration interaction between $d^{n}$ and $d^{n-1} s$ will be correctly accounted for by the Bacher and Goudsmit theory if the following relation exists betrieen the two perturbations (64) and (65):

$$
6 / 5 \Delta\left(d^{3}{ }^{2} \mathrm{G}\right)-3 / 7 \Delta\left(d^{2}{ }^{1} \mathrm{D}\right)=0 .
$$

According to the perturbation calculation used by Bacher and Goudsmit, the energy difference $W\left(\dot{d}^{3}\right.$ $\left.{ }^{2} \mathrm{G}\right)-W\left(d^{2} s{ }^{2} \mathrm{G}\right)$ would equal the difference $W\left(d^{2}\right.$ $\left.{ }^{1} \mathrm{D}\right)-W\left(d s^{1} \mathrm{D}\right)$ and the parameter $H_{2}\left(d^{3}-d^{2} s\right)$ would equal $H_{2}\left(d^{2}-d s\right)$. If these two conditions held, then (66) would clearly be satisfied. Relations similar to (66) can be found from other formulas (such as (29), (36), and (37)). By assuming that all energy differences $W\left(d^{n} \alpha S L\right)-W\left(d^{n-1} s \alpha^{\prime} S L\right)$ are equal, and all parameters $H_{2}\left(d^{n}-d^{n-1} s\right)$ are identical, matrix elements of configuration interaction can be calculated with the Bacher and Goudsmit theory by solving a set of simultaneous equations, although such a procedure would not be the most practical. Similar considerations would apply to any pair of configurations; in particular, the interaction between $d^{n} s$ and $d^{n-1} s^{2}$ is interesting because the parameter defined between these two configurations would be equal to the parameter defined between $d^{n}$ and $d^{n-1} s$ according to this direct application of the Bacher and Goudsmit theory.

Alternatively, (66) could be satisfied if the parameters $H_{2}\left(d^{3}-d^{2} s\right)$ and $H_{2}\left(d^{2}-d s\right)$ accidentally had the same ratio as the square roots of the energy denominators in (64) and (65). To get consistency for all formulas similar to (66) that could be derived, it is necessary that a representative average energy difference exist that approximates separations of terms with the same $S$ - and $L$-values in the two configurations (in many cases no average value will adequately represent the data). An easily defined value for this average is the difference in the $A$-values of the two configurations. In table 3 calculated $H_{2}$-values and differences between $A$-values have been collected. In a given atom, the differences between $A$-values in this table are greater the greater the ionization; $H_{2}$ is also larger in the spectra of higher ionization, according to the results obtained for vanadium.

An extension of this argument indicates that the polarization energy arising from interactions with configurations in which only one electron is excited may be partially included in the second approxima- 
TABLE 3. Average term separations and interaction parameters

\begin{tabular}{|c|c|c|c|c|c|c|c|c|}
\hline \multirow{2}{*}{ 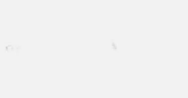 } & \multicolumn{2}{|c|}{ Titanium } & \multicolumn{2}{|c|}{ Vanadium } & \multicolumn{2}{|c|}{ Chromium } & \multicolumn{2}{|c|}{ Zirconium } \\
\hline & $A(\alpha)-A(\beta)$ & $H_{2}$ & $A(\alpha)-A(\beta)$ & $H_{2}$ & $A(\alpha)-A(\beta)$ & $H_{2}$ & $A(\alpha)-A(\beta)$ & $H_{2}$ \\
\hline$A(s)-A(d)$ & 80150 & & 147730 & & 227200 & & 37508 & \\
\hline $\begin{array}{l}A(d s)-A\left(d^{2}\right) \\
A\left(s^{2}\right)-A(d s)\end{array}$ & 34470 & & 92460 & & 162720 & & $\begin{array}{l}16700 \\
20304\end{array}$ & \\
\hline $\begin{array}{l}A\left(d^{2} s\right)-A\left(d^{3}\right) \\
H_{2} \\
A\left(d s^{2}\right)-A\left(d^{2} s\right)\end{array}$ & $\begin{array}{l}\text { a }-896 \\
\text { a } 16980\end{array}$ & a 172 & a 42102 & & 103100 & & $\begin{array}{r}\text { b }-1260 \\
\text { b } 3600\end{array}$ & b 387 \\
\hline $\begin{array}{l}A\left(d^{3} s\right)-A\left(d^{4}\right) \\
H_{2} \\
A\left(d^{2} s^{2}\right)-A\left(d^{3} s\right)\end{array}$ & $\begin{array}{r}-17140 \\
\mathrm{a}-13103\end{array}$ & & a 6332 & a 153 & 52424 & & $\begin{array}{l}-13230 \\
-11700\end{array}$ & \\
\hline $\begin{array}{l}A\left(d^{4} s\right)-A\left(d^{5}\right) \\
H_{2} \\
A\left(d^{3} s^{2}\right)-A\left(d^{4} s\right) \\
H_{2}\end{array}$ & & & $\begin{array}{l}c-21335 \\
\text { c }-8759\end{array}$ & c 106 & $\begin{array}{l}11875 \\
30400\end{array}$ & c 150 & & \\
\hline
\end{tabular}

a A. Many, Phys. Rev. 70, 511 (1946).

Rev. 14, $732(1933)$

c A. Schweizer, Phys. Rev. 80, 1080 (1950).

tion of BG. However, it is unlikely that the interaction parameters would behave exactly as required so that it might not be well accounted for.

It is clear from table 3 that in the titanium spectra of moderate ionization, the Bacher and Goudsmit method would not account for the configuration interaction. Even though the interaction is weak enough to be evaluated with second-order perturbation theory, the calculations discussed in section 5 show that errors of 300 to $700 \mathrm{~K}$ would be introduced into formulas (28), (29), (36), and (37). Whenever the average term separation is small or negative, which is the case in Ti $\mathrm{I}$ and Ti II, no set of values for the interaction parameters can produce agreement. When the configuration interaction is so strong that perturbation theory cannot be used, the errors are even larger. For zirconium, errors in excess of 1000 $\mathrm{K}$ result when the formulas are applied directly. The differences in $A$-values are all large and positive in chromium, so that the configuration interaction would probably be well accounted for in the second approximation; in this case, however, the interaction is weak and could be neglected in the linear theory.

\section{Irrational Roots}

This section deals with one of the simplest examples in which irrationality of the energy levels plays a part. As a preliminary, the two ${ }^{2} \mathrm{D}$ terms of the $d^{3}$ configuration are discussed.

The determination of the energy levels of the two ${ }^{2} \mathrm{D}$ terms of $d^{3}$ was first carried out by Ufford and Shortley. ${ }^{26}$ They chose two mutually orthogonal ${ }^{2} \mathrm{D}$ eigenfunctions, which they characterized as $a^{2} \mathrm{D}$ and $b^{2} \mathrm{D}$; in the more recent notation of III these same two eigenfunctions are designated as ${ }_{1}^{2} \mathrm{D}$ and ${ }_{3}^{2} \mathrm{D}$, respectively. ${ }^{27}$ In terms of this choice of eigenfunctions, the electrostatic energy matrix was evaluated

${ }^{26}$ C. W. Ufford and G. H. Shortley, Phys. Rev. 42, 167 (1932). See alsc, E. U. Condon and G. H. Shortley Theory of atomic spectra, p. 233 (Cambridge, 1951).

27 In III the classification of terms in $d^{n}$ is carried out by introducing the seniority number; this is given as the prefixed subscript in the notation above. and found to have the form

$$
M \equiv{ }_{3}^{2} \mathrm{D} D\left(\begin{array}{lc}
A+7 B+7 C & 3 \sqrt{21} B \\
3 \sqrt{21} B & A+3 B+3 C
\end{array}\right) .
$$

The energy eigenvalues of the two ${ }^{2} \mathrm{D}$ states of $d^{3}$ are the characteristic values of this matrix; these values (as well as the observed energies for the ${ }^{2} \mathrm{D}$ terms)

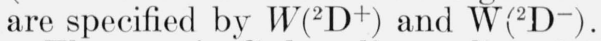

The matrix $S$ that diagonalizes (67) will have the form

$$
\begin{gathered}
S=\left(\begin{array}{r}
C_{1} C_{2} \\
-C_{2} C_{1}
\end{array}\right)\left(C_{1}^{2}+C_{2}^{2}=1\right), \\
S M S^{-1}=\left(\begin{array}{cc}
W\left({ }^{2} D^{+}\right) & 0 \\
0 & W\left({ }^{2} D^{-}\right)
\end{array}\right) .
\end{gathered}
$$

The elements of $S$ can be specified by the condition for the vanishing of the nondiagonal elements of $S M S^{-1}$

$\left[4 B\left(d^{3}\right)+4 C\left(d^{3}\right)\right] C_{1} C_{2}-\left(C_{1}^{2}-C_{2}^{2}\right) 3 \sqrt{21} B\left(d^{3}\right)=0$.

Although $W\left({ }_{1}^{2} \mathrm{D}\right)$ and $W\left({ }_{3}^{2} \mathrm{D}\right)$ have no physical reality, they will be used subsequently to indicate the energies that would be associated with the diagonal elements of the matrix (67). The following formulas are obtained from (69):

$$
\begin{aligned}
& W\left({ }_{3}^{2} \mathrm{D}\right)=C_{1}^{2} W\left({ }^{2} \mathrm{D}^{-}\right)+C_{2}^{2} W\left({ }^{2} \mathrm{D}^{+}\right), \\
& W\left({ }_{1}^{2} \mathrm{D}\right)=C_{1}^{2} W\left({ }^{2} \mathrm{D}^{+}\right)+C_{2}^{2} W\left({ }^{2} \mathrm{D}^{-}\right) .
\end{aligned}
$$

If the polarization energy can be accounted for with the $L(L+1)$ correction, then all the preceding discussion will still apply even when polarization energy is considered if the constant $A$ in (67) is suitably redefined.

By direct application of the methods in section 3, the energy for, say, a $d^{4}{ }^{3} \mathrm{G}$ term, can be expressed in 
the form

$$
\begin{aligned}
W_{2}\left(d^{4}{ }^{3} \mathrm{G}\right)^{*}= & 10 / 21 W\left(d^{3}{ }_{3}^{2} \mathrm{D}\right)^{*}+3 / 4 W\left(d^{3}{ }^{2} \mathrm{~F}\right)^{*} \\
& +4 / 3 W\left(d^{3}{ }^{4} \mathrm{~F}\right)+99 / 140 W\left(d^{3}{ }^{2} \mathrm{G}\right)^{*} \\
& +11 / 15 W\left(d^{3}{ }^{2} \mathrm{H}\right)-11 / 10 W\left(d^{2}{ }^{3} \mathrm{P}\right) \\
& -9 / 14 W\left(d^{2}{ }^{1} \mathrm{D}\right){ }^{*}-29 / 10 W\left(d^{2}{ }^{3} \mathrm{~F}\right) \\
& -19 / 14 W\left(d^{2}{ }^{1} \mathrm{G}\right)+4 W(d) .
\end{aligned}
$$

If the linear theory is valid, $W\left({ }_{3}^{2} \mathrm{D}\right)$ in $(73)$ can be replaced by the value (71); the approximation obtained in this way will be specified by a prime. In the exact application of the Bacher and Goudsmit theory, (71) can still be used to replace $W\left(d^{3}{ }_{3}^{2} \mathrm{D}\right)$, but $\dot{C}_{1}$ and $\mathrm{C}_{2}$ are not necessarily specified by $(70)$. Also, instead of evaluating $W\left(d^{3}{ }_{3}^{2} \mathrm{D} ; d^{2}\right)$ which was done in deriving (73), it is necessary to evaluate $\left.\bar{W}\left(d^{3} \mid \mathrm{C}_{1}{ }^{2} \mathrm{D}^{-}+C_{2}{ }^{2} \mathrm{D}^{+}\right] ; d^{2}\right)$. The following is the correct formula which is obtained in this way:

$$
\begin{aligned}
& W_{2}\left(d^{4}{ }^{3} \mathrm{G}\right)=W_{2}^{\prime}\left(d^{4}{ }^{3} \mathrm{G}\right)-\epsilon\left(d^{4}{ }^{3} \mathrm{G}\right) \\
\epsilon\left(d^{4}{ }^{3} \mathrm{G}\right)= & C_{1}^{2} C_{2}^{2}\left[8 / 21 W\left(d^{2}{ }^{1} \mathrm{~S}\right)-2 / 7 W\left(d^{2}{ }^{3} \mathrm{P}\right)\right. \\
& -50 / 147 W\left(d^{2}{ }^{1} \mathrm{D}\right)+2 / 7 W\left(d^{2}{ }^{3} \mathrm{~F}\right) \\
& \left.-2 / 49 W\left(d^{21} \mathrm{G}\right)\right]-\frac{C_{1} C_{2}\left(C_{1}^{2}-C_{2}^{2}\right)}{14 \sqrt{21}}\left[21 W\left(d^{2}{ }^{3} \mathrm{P}\right)\right. \\
& \left.-15 W\left(d^{2}{ }^{1} \mathrm{D}\right)-21 W\left(d^{2}{ }^{3} \mathrm{~F}\right)+15 W\left(d^{2}{ }^{1} \mathrm{G}\right)\right] .
\end{aligned}
$$

Replacing the $d^{2}$ energies by their Slater formulas (including the $L(L+1)$ correction) leads to the following approximate form for $(75)$ :

$$
\begin{aligned}
\epsilon\left(d^{4}{ }^{3} \mathrm{G}\right)= & 10 / 21 C_{1} C_{2}\left\{\left[4 B\left(d^{2}\right)+4 C\left(d^{2}\right)\right] C_{1} C_{2}-\left(C_{1}^{2}\right.\right. \\
& \left.\left.-C_{2}^{2}\right) 3 \sqrt{21} B\left(d^{2}\right)\right\}
\end{aligned}
$$

It follows that when $C_{1}$ and $C_{2}$ are determined from (70) then (74) will be equivalent to (73) if the ratio of $B$ to $C$ is the same in $d^{3}$ as it is in $d^{2}$. To establish the general correctness of the method of applying the Bacher and Goudsmit theory which was developed in section 5, it is necessary that in general the ratios of similarly defined radial parameters be the same in all spectra of a given atom.

It is well known that the ratio of $B$ to $C$ is very nearly constant. Values of this ratio, obtained mainly from the calculations discussed in section 5, are given in table 4 . Because of the large errors that may be present in the theory $( \pm 400 \mathrm{~K})$, the values of $r_{2}$, obtained by fitting the observed data exactly, are not well defined; to a lesser extent the $d^{3}$ and $d^{3} s$ configurations also have too few terms to define $r_{3}$ precisely. The data as a whole (giving strong weight to the two well determined $r_{5}$-values) support the conclusion that $r_{n}$ is a slowly decreasing function of $n$. The exception apparent in titanium is less noticeable if the irrational roots are omitted from the least-squares calculations for $3 d^{3}$ and $3 d^{3} 4 s$ parameters (the error in fitting the irrational terms is then excessive, however).

To estimate roughly the effect that could result from inequality of this ratio, (76) can be put in the following form with the help of (70), and by use of an approximate value of $r$ equal to 0.29 :

$$
\begin{aligned}
\epsilon\left(d^{4}{ }^{3} \mathrm{G}\right) & =40 / 21\left(\frac{r_{3}-r_{2}}{r_{3}}\right) C_{1}^{2} C_{2}^{2} C\left(d^{2}\right) \\
& =1.16\left(r_{3}-r_{2}\right) C\left(d^{2}\right) .
\end{aligned}
$$

\begin{tabular}{|c|c|c|c|}
\hline & $r_{2}$ & $r_{3}$ & $r_{5}$ \\
\hline $\begin{array}{ll}\mathrm{Ti} & 1 \\
\mathrm{Ti} & \text { II } \\
\mathrm{Ti} & \text { III } \\
\mathrm{Fe} & \text { III a }\end{array}$ & $\begin{array}{r}0.289 \\
.290 \\
.291\end{array}$ & $\begin{array}{r}\text { c } 0.313(0.319) \\
.317(0.290)\end{array}$ & 0.271 \\
\hline $\begin{array}{ll}\mathrm{Zr} & \text { I } \\
\mathrm{Zr} & \text { II } \\
\mathrm{Zr} & \text { III } \\
\text { Mo I } & \text { b }\end{array}$ & $\begin{array}{l}.304 \\
.289 \\
.297\end{array}$ & $\begin{array}{l}.279 \\
.287\end{array}$ & .257 \\
\hline
\end{tabular}

In Ti III, $C\left(d^{2}\right)=2505$ so that if $r_{3}-r_{2}=0.05$ then $\epsilon=145 \mathrm{~K}$. This is likely to overestimate the maximum error that can result, yet within the accuracy of the theory it probably could be neglected. However, the same treatment for the $d^{4}{ }^{1} \mathrm{~F}$ term shows that $\epsilon$ will have exactly 3 times the value $(75)$, and for the $d^{4}{ }^{3} \mathrm{D}$ term the error will be 2.4 times this value, so it is not certain that this effect will always be negligible. The ratio $G_{2} / C$ need not be constant (it varies considerably, from 0.4 to 0.8 usually).

TABLE 4. B/C ratios

$r_{n}$ is the ratio of $R\left(d^{n}\right)$ to $C\left(d^{n}\right), R\left(d^{n} s\right)$ to $C\left(d^{n} s\right)$, or $R\left(d^{n} s^{2}\right)$ to $C\left(d^{n} s^{2}\right)$, accord ing to whichever definition has meaning for the spectrum specified.

a See footnote 8 , page 38 b See footnote 3 , page 35 . - Values in parentheses are calculated with irrational roots omitted.

Though the method of BG introduces new nonlinear effects if the parameter ratios are not equal, these effects would probably represent errors in the method. Because of this, and because the ratios are so nearly equal, it is not expected that a correction with this variation will produce improved agreement when added to the linear theory. However, irrational terms often cannot be fitted by the linear theory with the accuracy that is obtainable for rational terms ${ }^{28}$ and some additional correction of this form may be needed. This correction would not change the agreement obtainable for the rational terms of $d^{4}$, but it would introduce corrections into the irrational terms. These considerations may be less academic for spectra with $p$ - and $d$-electrons where there are more parameter ratios that may differ, and where most of the terms are irrational. ${ }^{29}$

I am indebted to C. C. Kiess for suggesting the problem on which this work is based, and I thank also G. Racah and C. W. Ufford for their comments on the original manuscript of this paper.

\footnotetext{
${ }^{28}$ R. E. Trees, Phys. Rev. 85, 382 (1952).

${ }_{29}$ Meshkov has introduced a method which combines the Slater theory with a Bacher and Goudsmit first approximation, and has obtained improved agreement for the $3 d^{2} 4 p$ configuration of Ti II (footnote 18). A simple extension of the linear theory to include correlations between $p$ - and $d$-electrons may explain this improvement, but the possibility that new types of crorections are needed to explain it, cannot vet be excluded. His theory also leads to better agreement than is obtainable with the Slater theory alone, in the $d^{3}$ configuration of $\mathrm{V}$ II and the $d^{4}$ configuration of V II (Phys. Rev. 93, 270 (1954)). In the $d^{4}$ configur and the $d^{4}$ configuration of $\mathrm{II}$ (Phys. Rev. 93, 2 $(1954)$. In the $d^{\prime}$ configura tion his final mean deviation of $\pm 663 \mathrm{~K}$ is still rather large; this may be because there are a large number of irrational terms present in this configuration or
because configuration interaction was neglected. See also S. Meshkov and $\mathrm{C}$. W. Ufford, Phys. Rev. 94, 75 (1954).

Washington, January 8, 1954.
} 